\begin{tabular}{|c|l|}
\hline Title & $\begin{array}{l}\text { A DETERMINISTIC GAME INTERPRETA TION FOR FULLY NONLINEAR PARA BOLIC EQUA TIONS WITH } \\
\text { DYNAMIC BOUNDARY CONDITIONS }\end{array}$ \\
\hline Author(s) & HAMAMUKI, NA O; LIU, QING \\
\hline Citation & Hokkaido University Preprint Series in Mathematics, 1123, 1-42 \\
\hline Issue Date & 2019-03-19 \\
\hline DOI & 10.14943/87676 \\
\hline Doc URL & http://hdl.handle.net/2115/73100 \\
\hline Type & bulletin (article) \\
\hline File Information & DeterministicGame.pdf \\
\hline
\end{tabular}

Instructions for use 


\title{
A DETERMINISTIC GAME INTERPRETATION FOR FULLY NONLINEAR PARABOLIC EQUATIONS WITH DYNAMIC BOUNDARY CONDITIONS
}

\author{
NAO HAMAMUKI AND QING LIU
}

\begin{abstract}
This paper is devoted to deterministic discrete game-theoretic interpretations for fully nonlinear parabolic and elliptic equations with nonlinear dynamic boundary conditions. It is known that the classical Neumann boundary condition for general parabolic or elliptic equations can be generated by including reflections on the boundary to the interior optimal control or game interpretations. We study a dynamic version of such type of boundary problems, generalizing the discrete game-theoretic approach proposed by Kohn-Serfaty $(2006,2010)$ for Cauchy problems and later developed by Giga-Liu (2009) and Daniel (2013) for Neumann type boundary problems.
\end{abstract}

\section{INTRODUCTION}

In this paper, we are interested in a deterministic discrete game-theoretic interpretation for fully nonlinear parabolic equations with dynamic boundary conditions. We mainly consider boundary value problems of the form

$$
\begin{cases}\partial_{t} u+F\left(x, \nabla u, \nabla^{2} u\right)=0 & \text { in } \Omega \times(0, \infty), \\ \partial_{t} u+H(x, \nabla u)=0 & \text { on } \partial \Omega \times(0, \infty), \\ u(\cdot, 0)=u_{0} & \text { in } \bar{\Omega} .\end{cases}
$$

We below give a brief description of the notations appearing in (DBP1):

- $\Omega \subset \mathbb{R}^{n}$ is a bounded domain with boundary of $C^{2}$ class. Let $\nu(x)$ denote the unit outward normal to $\partial \Omega$ at $x \in \partial \Omega$.

- $u_{0}: \bar{\Omega} \rightarrow \mathbb{R}$ denotes a given Lipschitz function.

- $F: \bar{\Omega} \times \mathbb{R}^{n} \times \mathbb{S}^{n} \rightarrow \mathbb{R}$ is a continuous fully nonlinear elliptic operator satisfying appropriate assumptions to be elaborated later. Here $\mathbb{S}^{n}$ stands for the set of all $n \times n$ real symmetric matrices.

- $H: \partial \Omega \times \mathbb{R}^{n} \rightarrow \mathbb{R}$ denotes a general continuous boundary operator. A typical example of $H$ is

$$
H(x, p)=\langle\nu(x), p\rangle
$$

for all $x \in \partial \Omega$ and $p \in \mathbb{R}^{n}$, where $\langle\cdot, \cdot\rangle$ stands for the inner product in $\mathbb{R}^{n}$; in other words, the boundary condition (1.2) in this case reduces to

$$
\partial_{t} u+\langle\nu(x), \nabla u\rangle=0 \quad \text { on } \partial \Omega \times(0, \infty) .
$$

It is possible to consider a more general class of boundary Hamiltonian $H$, which will be introduced in a moment.

Date: March 18, 2019.

2010 Mathematics Subject Classification. 35K61, 35J66, 35Q91, 35D40.

Key words and phrases. dynamic boundary problems, discrete differential games, viscosity solutions. 
We remark that by the boundedness and $C^{2}$ regularity of $\Omega, \nu$ can be extended to a Lipschitz function $\bar{\Omega} \rightarrow B_{1}(0)$, where $B_{r}(x)$ denotes the closed ball centered at $x$ with radius $r>0$.

A comparison principle for (DBP1) in the framework of viscosity solutions is established in $[7,8]$. In Section 3.2 , we review the definition of viscosity solutions to (DBP1) and comparison results.

We aim to construct a family of discrete deterministic two-person games whose value functions approximate the viscosity solution of (DBP1), inspired by the pioneer work [35] on such game interpretations for Cauchy problems. Our present work will also serve as an existence result for (DBP1). We stress that it is possible to extend our results to a more general class of fully nonlinear equations with $F$ depending also on the time variable $t$ and the unknown function $u$, but we prefer to stick to the current special form since our main concern lies at the dynamic boundary condition.

1.1. Background and motivation. Before proceeding to our main results, let us briefly review the related literature and state the motivation of our work. Dynamic boundary conditions appear in research work for various parabolic equations with important applications; see for instance [18, 15, 49, 27, 48, 12]. We refer to [17, 19, 2, 26, 22, 24, 23] etc. for study on elliptic equations with dynamic boundary conditions.

In the context of viscosity solutions, as mentioned above, a comparison principle is provided in $[7,8]$ for a general class of fully nonlinear parabolic equations with dynamic boundary conditions. Motivated by applications in superconductivity and interface evolution, a class of dynamic boundary problems for the Hamilton-Jacobi equations are studied in [16] when the boundary Hamiltonian $H$ is independent of $\nabla u$. More recently, existence and uniqueness for mean curvature flow equation with the linear dynamic boundary condition (1.4) in a half space are established in [29].

Besides the wellposedness results, asymptotic behavior for the Hamilton-Jacobi equations with dynamic boundary conditions is studied in $[9,1]$. The authors of [1] study asymptotic behavior of solutions to a dynamic problem for the eikonal equation

$$
\varepsilon \partial_{t} u+|\nabla u|=1 \quad \text { in } \Omega \times(0, \infty)
$$

as $\varepsilon \rightarrow 0$. Such type of asymptotics is recently discussed in [25] for the heat equation.

As for the connection between nonlinear PDEs and discrete games, we recall that a deterministic game-based approach is proposed by Kohn and Serfaty in [34] for the mean curvature flow equation and in [35] for general parabolic and elliptic equations. We also refer to the work by Peres et al. $[43,44]$ on stochastic discrete games called Tug-ofwar for $p$-Laplace equations with $1<p \leq \infty$; see related results in [41, 40]. The game approximations turn out to be useful in understanding various analytic and geometric properties of the associated nonlinear PDEs, as shown in $[4,37,39,38,45,42]$ etc.

However, the games mentioned above are all constructed for nonlinear equations either in the whole space or in a domain with Dirichlet boundary conditions. A continuoustime stochastic interpretation for Neumann boundary problems is provided in [36]. In the spirit of $[34,35]$, deterministic discrete game interpretations of the Neumann type boundary problems for curvature flow equations and more general parabolic equations are studied respectively in [30] and in [14] by including reflected or projected dynamics on the boundary in the games; see also $[11,3]$ for stochastic discrete games associated to the infinity Laplacian. 
Despite the above development of the game-theoretic approach, it was not clear if the game method can be generalized for nonlinear dynamic boundary conditions. The present work attempts to answer this question and is devoted to an extension of the results in $[35,30,14]$ to a general class of fully nonlinear dynamic boundary conditions. In [31] we study the game interpretation for dynamic boundary problem of level set curvature flow equation and discuss several applications of the game method.

1.2. Assumptions. We first impose some basic assumptions on the operator $F$ in (1.1):

(F1) $F$ is a continuous (degenerate) elliptic operator locally Lipschitz in $p$ and $X$; namely, for any $R>0$, there exists $L_{F}(R)>0$ such that

$$
\left|F(x, p, X)-F\left(x, p^{\prime}, X^{\prime}\right)\right| \leq L_{F}(R)\left(\left|p-p^{\prime}\right|+\left|X-X^{\prime}\right|\right)
$$

for all $x \in \bar{\Omega}, p, p^{\prime} \in \mathbb{R}^{n}, X, X^{\prime} \in \mathbb{S}^{n}$ with $|p|+\left|p^{\prime}\right|+|X|+\left|X^{\prime}\right| \leq R$, and

$$
F(x, p, X) \leq F\left(x, p, X^{\prime}\right)
$$

for any $x \in \bar{\Omega}, p \in \mathbb{R}^{n}$ and $X, X^{\prime} \in \mathbb{S}^{n}$ satisfying $X \geq X^{\prime}$.

(F2) There exist $C>0, \sigma_{1}, \sigma_{2}>0$ such that

$$
|F(x, p, X)| \leq C\left(1+|p|^{\sigma_{1}}+|X|^{\sigma_{2}}\right) \quad \text { for all } x \in \bar{\Omega}, p \in \mathbb{R}^{n} \text { and } X \in \mathbb{S}^{n} .
$$

Let us next introduce a general nonlinear boundary Hamiltonian $H$ in (1.2). Let $A$ be a compact metric space. For later use, we take for every $\lambda>0$

$$
\Omega_{\lambda}=\{x \in \Omega: \operatorname{dist}(x, \partial \Omega)>\lambda\} .
$$

For any $a \in A$, we denote by $\gamma_{a}(x)$ an outward unit oblique normal to $\partial \Omega$.

Throughout this work we assume that

(H1) The oblique normal $\gamma_{a}$ can be extended to a vector-valued function $\bar{\Omega} \rightarrow B_{1}(0)$ that is Lipschitz continuous uniformly for all $a \in A$.

(H2) There exists a uniform positive lower bound for $\left\langle\gamma_{a}, n\right\rangle$ on $\partial \Omega$; namely,

$$
m=\inf _{\substack{a \in A \\ x \in \partial \Omega}}\left\langle\gamma_{a}(x), \nu(x)\right\rangle>0 .
$$

The assumption (H1) is satisfied when $\gamma_{a}=\nu$ on $\partial \Omega$ for all $a \in A$. In this case, we can choose a Lipschitz extension of $\nu$ in $\bar{\Omega}$ such that $\nu(x)=-\nabla d(x)$ for any $x \in \bar{\Omega} \backslash \Omega_{\lambda_{0}}$ with $\lambda_{0}>0$ small, where $d(x)$ denotes the signed distance from $x$ to $\partial \Omega$, i.e.,

$$
d(x)=\operatorname{dist}\left(x, \Omega^{c}\right)-\operatorname{dist}(x, \Omega) .
$$

Note that $d$ is of class $C^{2}$ near $\partial \Omega$ due to the $C^{2}$ regularity of $\Omega$. The Lipschitz extension in $\Omega_{\lambda_{0}}$ can be done following [20, Page 80].

Suppose that $H: \bar{\Omega} \times \mathbb{R}^{n} \rightarrow \mathbb{R}$ is given in the form

$$
H(x, p)=\min _{a \in A}\left\{\left\langle p, \gamma_{a}(x)-f(x, a)\right\rangle+g(x, a)\right\},
$$

where $f, g: \bar{\Omega} \times A \rightarrow \mathbb{R}$ satisfy the following assumptions:

(B1) $f, g$ are both bounded and Lipschitz in $\bar{\Omega} \times A$. 
(B2) The function $f$ satisfies

$$
\sup _{(x, a) \in \bar{\Omega} \times A}|f(x, a)|<m
$$

where $m>0$ is given in (1.7).

The assumption (B1) together with (H1) implies the existence of a constant $L_{H}>0$ fulfilling

$$
|H(x, p)-H(y, q)| \leq L_{H}((1+|p|+|q|)|x-y|+|p-q|)
$$

for all $x, y \in \bar{\Omega}$ and $p, q \in \mathbb{R}^{n}$. Moreover, (B2) combined with (H2) implies that there exists $\rho>0$ such that for any $x \in \bar{\Omega}$ and $p \in \mathbb{R}^{n}$,

$$
H(x, p+s \nu(x))-H(x, p) \geq \rho s \quad \text { for all } s>0 .
$$

In fact, we may choose

$$
\rho=m-\sup _{(x, a) \in \bar{\Omega} \times A}|f(x, a)|>0
$$

to obtain (1.11). This amounts to saying that in (1.2) the classical Neumann part $\left\langle\nabla u, \gamma_{a}\right\rangle$ plays a nontrivial role uniformly for all $a \in A$. This is not only important for our game interpretation but also for uniqueness of viscosity solutions to (DBP1); we refer to $[7,8]$ for a comparison principle that essentially requires this property.

It is clear that the linear boundary condition (1.4) satisfies (B1) and (B2). A typical example of nonlinear dynamic boundary conditions is

$$
\partial_{t} u+\langle\nabla u, \nu(x)\rangle=K|\nabla u|+g(x) \text { on } \partial \Omega \times(0, \infty)
$$

with any $0 \leq K<1$, for which we take in (1.8) $A=B_{1}(0), \gamma_{a} \equiv \nu, f(x, a) \equiv K a$ and $g(x, a) \equiv g(x)$.

1.3. The game setting and main result. Let us now provide a game interpretation for the general dynamic boundary problem (DBP1).

In order to generate the dynamic boundary condition (1.2), we need to prepare a "cushion" near the boundary $\partial \Omega$. Let $\alpha, \beta, \gamma \in(0,1)$ satisfy several assumptions to be precisely given later. Take $0<\mu<1-\gamma$. We fix a step size $\varepsilon>0$ and set $N=\left[t / \varepsilon^{2}\right]$ for any given $(x, t) \in \bar{\Omega} \times[0, \infty)$. We next set, for every $x \in \bar{\Omega}$,

$$
\eta_{\varepsilon}(x)=\min \left\{1, \frac{\operatorname{dist}(x, \partial \Omega)}{\varepsilon^{\mu}}\right\}
$$

and take

$$
\zeta_{\varepsilon}(x)=1-\eta_{\varepsilon}^{2}(x)
$$

Let us start the game from $y_{0}=x \in \bar{\Omega}$. At the $k$-th step $(k=1,2, \ldots, N)$,

- Player I chooses $p_{k} \in \mathbb{R}^{n}, X_{k} \in \mathbb{S}^{n}$ satisfying $\left|p_{k}\right| \leq \varepsilon^{-\alpha}$ and $\left|X_{k}\right| \leq \varepsilon^{-\beta}$;

- Player II then picks $a_{k} \in A$ and $v_{k} \in \mathbb{R}^{n}$ with $\left|v_{k}\right| \leq \varepsilon^{-\gamma}$;

- Once the choices of both players are determined, the game position moves from $y_{k}$ to a new point $y_{k}=y_{k-1}+h_{k}$ (to be proved to belong to $\bar{\Omega}$ later for $\varepsilon$ sufficiently small), where

$$
h_{k}=\sqrt{2} \varepsilon \eta_{\varepsilon}\left(y_{k-1}\right) v_{k}+\varepsilon^{2} \zeta_{\varepsilon}\left(y_{k-1}\right)\left(f\left(y_{k-1}, a_{k}\right)-\gamma_{a_{k}}\left(y_{k-1}\right)\right)
$$

and $\eta_{\varepsilon}, \zeta_{\varepsilon}$ are the functions given respectively in (1.12) and (1.13). 
- Meanwhile, Player II pays the following amount of money to Player I:

$$
\begin{aligned}
l_{k}=\sqrt{2} \varepsilon \eta_{\varepsilon}\left(y_{k-1}\right)\left\langle p_{k}, v_{k}\right\rangle+\varepsilon^{2} \eta_{\varepsilon}^{2}\left(y_{k-1}\right) & \left(\left\langle X_{k} v_{k}, v_{k}\right\rangle+F\left(y_{k-1}, p_{k}, X_{k}\right)\right) \\
+ & \varepsilon^{2} \zeta_{\varepsilon}\left(y_{k-1}\right) g\left(y_{k-1}, a_{k}\right) .
\end{aligned}
$$

The rules above define a sequence of positions $y_{0}(=x), y_{1}, y_{2}, \ldots, y_{N}$. When the game ends after the $N$-th round, Player II receives from Player I a terminal fee $u_{0}\left(y_{N}\right)$. The game outcome for Player II at $(x, t)$, determined by $p_{k}, X_{k}, a_{k}, v_{k}$ for $k=1, \ldots, N$, is therefore

$$
J^{\varepsilon}(x, t)=u_{0}\left(y_{N}\right)-\sum_{k=1}^{N} l_{k} .
$$

Suppose that Player II attempts to maximize $J^{\varepsilon}(x, t)$ while Player I is to minimize the same amount. We thus can define the value function as

$$
u^{\varepsilon}(x, t)=\min _{p_{1}, X_{1}} \max _{a_{1}, v_{1}} \min _{p_{2}, X_{2}} \max _{a_{2}, v_{2}} \ldots \min _{p_{N}, X_{N}} \max _{a_{N}, v_{N}} J^{\varepsilon}(x, t) .
$$

Let us now verify that $y_{k-1}+h_{k} \in \bar{\Omega}$ for all $k=1,2, \ldots, N$ when $\varepsilon>0$ is sufficiently small. To see this, we omit all of the sub-indices of $y_{k-1}, h_{k}, a_{k}$ for simplicity of notation. Then we have

$$
\operatorname{dist}\left(y+h, \Omega^{c}\right)=\operatorname{dist}\left(z+\sqrt{2} \varepsilon \eta_{\varepsilon}(y) v, \Omega^{c}\right),
$$

where

$$
z=y+\varepsilon^{2} \zeta_{\varepsilon}(y)\left(f(y, a)-\gamma_{a}(y)\right) .
$$

Thanks to (H2) and the $C^{2}$ regularity of $\Omega$, we get

$$
\operatorname{dist}\left(y-\varepsilon^{2} \zeta_{\varepsilon}(y) \gamma_{a}(y), \Omega^{c}\right) \geq \operatorname{dist}\left(y, \Omega^{c}\right)+m \varepsilon^{2} \zeta_{\varepsilon}(y)+o\left(\varepsilon^{2} \zeta_{\varepsilon}(y)\right),
$$

which, by (B2), implies that

$$
\operatorname{dist}\left(z, \Omega^{c}\right) \geq \operatorname{dist}\left(y, \Omega^{c}\right)+\varepsilon^{2} \zeta_{\varepsilon}(y)\left(m-\sup _{\bar{\Omega} \times A}|f| \varepsilon^{2}\right)+o\left(\varepsilon^{2} \zeta_{\varepsilon}(y)\right) \geq \operatorname{dist}\left(y, \Omega^{c}\right)
$$

when $\varepsilon$ is taken small without depending on $y$ and $\bar{\Omega}$. In view of (1.12), it follows that

$$
\operatorname{dist}\left(z+\sqrt{2} \varepsilon \eta_{\varepsilon}(y) v, \Omega^{c}\right) \geq \operatorname{dist}\left(z, \Omega^{c}\right)-\sqrt{2} \varepsilon \eta_{\varepsilon}(y)|v| \geq \operatorname{dist}\left(y, \Omega^{c}\right)\left(1-\sqrt{2} \varepsilon^{1-\gamma-\mu}\right) \geq 0
$$

when $\varepsilon>0$ is taken further small if necessary.

We remark that although the game dynamics and cost functions look complicated, the connection with equations can somehow be easily observed; all of the terms involving $\eta_{\varepsilon}$ are used to derive the equation (1.1) in the interior while the terms related to the boundary condition (1.2) are all carrying $\zeta_{\varepsilon}$.

As an immediate consequence of (1.16), the so-called dynamic programming principle in this case reads

$$
u^{\varepsilon}(x, t)=\min _{p, X} \max _{a, v}\left\{u^{\varepsilon}\left(x+h, t-\varepsilon^{2}\right)-l\right\},
$$

where $|p| \leq \varepsilon^{-\alpha},|X| \leq \varepsilon^{-\beta}, a \in A$ and $|v| \leq \varepsilon^{-\gamma}$, and

$$
\begin{gathered}
h=\sqrt{2} \varepsilon \eta_{\varepsilon}(x) v+\varepsilon^{2} \zeta_{\varepsilon}(x)\left(f(x, a)-\gamma_{a}(x)\right), \\
l=\sqrt{2} \varepsilon \eta_{\varepsilon}(x)\langle p, v\rangle+\varepsilon^{2} \eta_{\varepsilon}^{2}(x)(\langle X v, v\rangle+F(x, p, X))+\varepsilon^{2} \zeta_{\varepsilon}(x) g(x, a) .
\end{gathered}
$$


We next list the assumptions on the choice of $\alpha, \beta, \gamma$ in order to rigorously show the convergence of $u^{\varepsilon}$ in the interior, as used also in [35, 14]:

$$
\begin{gathered}
\gamma<\frac{1}{3}, \\
\alpha+\gamma<1, \quad \beta+2 \gamma<2, \quad \max \left\{\alpha \sigma_{1}, \beta \sigma_{2}\right\}<2, \\
\beta<1-\gamma, \quad \beta \sigma_{2}<1+\gamma, \\
\alpha\left(\sigma_{1}-1\right)<\gamma+1, \\
\beta\left(\sigma_{2}-1\right)<2 \gamma .
\end{gathered}
$$

We remark that (1.21) is utilized to guarantee the smallness of $\varepsilon\langle p, v\rangle, \varepsilon^{2}\langle X v, v\rangle$ and $\varepsilon^{2} F(x, p, X)$ respectively so that the running cost $l \rightarrow 0$ as $\varepsilon \rightarrow 0$.

In order to state our main result, let us take the relaxed half limits of $u^{\varepsilon}$, as in [13] for example:

$$
\begin{aligned}
& \bar{u}(x, t):=\lim _{\delta \rightarrow 0} \sup \left\{u^{\varepsilon}(y, s):(y, s) \in \bar{\Omega} \times[0, \infty),|x-y|+|t-s|+\varepsilon \leq \delta\right\}, \\
& \underline{u}(x, t):=\lim _{\delta \rightarrow 0} \inf \left\{u^{\varepsilon}(y, s):(y, s) \in \bar{\Omega} \times[0, \infty),|x-y|+|t-s|+\varepsilon \leq \delta\right\} .
\end{aligned}
$$

It is clear that $\bar{u} \in U S C(\bar{\Omega} \times[0, \infty))$ and $\underline{u} \in \operatorname{LSC}(\bar{\Omega} \times[0, \infty))$.

Theorem 1.1 (Game approximation for general dynamic boundary problems). Assume that $\Omega \subset \mathbb{R}^{n}$ is a bounded $C^{2}$ domain. Assume that (H1), (H2), (F1), (F2), (B1), (B2) hold. Let $H$ be given by (1.8). Assume that $u_{0}$ is Lipschitz in $\bar{\Omega}$. For any $\varepsilon>0$ small, let $u^{\varepsilon}$ be the value function defined as in (1.16) under the conditions (1.20)-(1.24). Then $\bar{u}$ and $\underline{u}$ given in (1.25) are respectively a subsolution and a supersolution of (1.1)-(1.2) with $\bar{u}(\cdot, 0)=u_{0}=\underline{u}(\cdot, 0)$ in $\bar{\Omega}$. Moreover, if the comparison principle for (DBP1) holds, then $u^{\varepsilon} \rightarrow u$ locally uniformly in $\bar{\Omega} \times[0, \infty)$ as $\varepsilon \rightarrow 0$, where $u$ is a unique solution of (DBP1).

It is possible to obtain a similar game interpretation for parabolic equations with boundary conditions more general than (1.8); see Section 3.2 for details.

1.4. A heuristic proof of game convergence. Let us give a heuristic proof of Theorem 1.1 in what follows. The rigorous proof is presented in detail in Section 3.1. Suppose that the value function $u^{\varepsilon}$ is smooth in $\bar{\Omega} \times(0, \infty)$. Applying Taylor expansion to the dynamic programming equation (1.17), we have at any $(x, t) \in \bar{\Omega} \times(0, \infty)$

$$
0=\min _{p, X} \max _{a, v}\left\{\left\langle\nabla u^{\varepsilon}, h\right\rangle+\frac{1}{2}\left\langle\nabla^{2} u^{\varepsilon} h, h\right\rangle-\varepsilon^{2} \partial_{t} u^{\varepsilon}-l\right\}+o\left(\varepsilon^{2}\right),
$$

where $h$ and $l$ are given as in (1.18) and (1.19). Here we used (1.20) to formally deduce that $|h|^{3}$ is of order $o\left(\varepsilon^{2}\right)$.

The derivation of the equation (1.1) follows the same argument as in [35], since $\eta_{\varepsilon}(x) \rightarrow$ 1 and $\zeta_{\varepsilon}(x) \rightarrow 0$ as $\varepsilon \rightarrow 0$ for any $x \in \Omega$. We thus focus our attention only to the case when $x \in \partial \Omega$. In order to imitate our rigorous proof in this case, we assume that there 
exists an approximating sequence of any $(x, t) \in \partial \Omega \times(0, \infty)$, denoted by $\left(x_{\varepsilon}, t_{\varepsilon}\right)$, where (1.26) holds. Then we have at $\left(x_{\varepsilon}, t_{\varepsilon}\right)$

$$
\begin{aligned}
\varepsilon^{2} \partial_{t} u^{\varepsilon}=\min _{p, X} \max _{v}\left\{\sqrt{2} \varepsilon \eta_{\varepsilon}\left\langle\nabla u^{\varepsilon}-p, v\right\rangle+\varepsilon^{2} \eta_{\varepsilon}^{2}\left(\left\langle\left(\nabla^{2} u^{\varepsilon}-X\right) v, v\right\rangle-F\left(x_{\varepsilon}, p, X\right)\right)\right\} \\
+\max _{a}\left\{\varepsilon^{2} \zeta_{\varepsilon}\left\langle\nabla u^{\varepsilon}, f\left(x_{\varepsilon}, a\right)-\gamma_{a}\right\rangle-\varepsilon^{2} \zeta_{\varepsilon} g\left(x_{\varepsilon}, a\right)\right\}+o\left(\varepsilon^{2}\right) .
\end{aligned}
$$

Our following argument for the first part on the right hand relies on the choices of $\alpha, \beta$ and $\gamma$ as in (1.22)-(1.24). Roughly speaking, due to the maximizing variable $v$, the minimum needs to be attained approximately at $p=\nabla u^{\varepsilon}\left(x_{\varepsilon}, t_{\varepsilon}\right)$ and a certain $X \geq \nabla^{2} u^{\varepsilon}\left(x_{\varepsilon}, t_{\varepsilon}\right)$ due to the lowest order term $\sqrt{2} \varepsilon \eta_{\varepsilon}\left\langle\nabla u^{\varepsilon}-p, v\right\rangle$ and then the second dominant term $\varepsilon^{2} \eta_{\varepsilon}^{2}\left\langle\left(\nabla^{2} u^{\varepsilon}-X\right) v, v\right\rangle$.

Under such choices of $p$ and $X,|v|$ can be taken small. By the ellipticity of $F$, we thus have

$$
\begin{gathered}
\min _{p, X} \max _{v}\left\{\sqrt{2} \varepsilon \eta_{\varepsilon}\left\langle\nabla u^{\varepsilon}-p, v\right\rangle+\varepsilon^{2} \eta_{\varepsilon}^{2}\left(\left\langle\left(\nabla^{2} u^{\varepsilon}-X\right) v, v\right\rangle-F\left(x_{\varepsilon}, p, X\right)\right)\right\} \\
=-\varepsilon^{2} \eta_{\varepsilon}^{2} F\left(x_{\varepsilon}, \nabla u^{\varepsilon}, \nabla^{2} u^{\varepsilon}\right)+o\left(\varepsilon^{2}\right) .
\end{gathered}
$$

On the other hand, in view of (1.8), we have

$$
\max _{a}\left\{\varepsilon^{2} \zeta_{\varepsilon}\left(x_{\varepsilon}\right)\left\langle\nabla u^{\varepsilon}\left(x_{\varepsilon}, t_{\varepsilon}\right), f\left(x_{\varepsilon}, a\right)-\gamma_{a}\right\rangle-\varepsilon^{2} \zeta_{\varepsilon} g\left(x_{\varepsilon}, a\right)\right\}=-\varepsilon^{2} \zeta_{\varepsilon} H\left(x_{\varepsilon}, \nabla u^{\varepsilon}\left(x_{\varepsilon}, t_{\varepsilon}\right)\right) .
$$

Combined with (1.27) and (1.28), we are then led to

$$
\varepsilon^{2} \partial_{t} u^{\varepsilon}+\varepsilon^{2} \eta_{\varepsilon}^{2} F\left(x_{\varepsilon}, \nabla u^{\varepsilon}, \nabla^{2} u^{\varepsilon}\right)+\varepsilon^{2} \zeta_{\varepsilon} H\left(\nabla u^{\varepsilon}, \nabla^{2} u^{\varepsilon}\right)=o\left(\varepsilon^{2}\right)
$$

at $\left(x_{\varepsilon}, t_{\varepsilon}\right)$, which is equivalent to

$$
\partial_{t} u^{\varepsilon}+\eta_{\varepsilon}^{2} F\left(x_{\varepsilon}, \nabla u^{\varepsilon}, \nabla^{2} u^{\varepsilon}\right)+\zeta_{\varepsilon} H\left(x_{\varepsilon}, \nabla u^{\varepsilon}\right)=o(1) .
$$

Recalling that $\eta_{\varepsilon}^{2}\left(x_{\varepsilon}\right)+\zeta_{\varepsilon}\left(x_{\varepsilon}\right)=1$ for all $\varepsilon>0$ as in (1.13), we may take a subsequence such that $\eta_{\varepsilon}^{2}\left(x_{\varepsilon}\right) \rightarrow c$ with $0 \leq c \leq 1$ as $\varepsilon \rightarrow 0$. It follows that at $(x, t) \in \partial \Omega \times(0, \infty)$,

$$
c\left(\partial_{t} u+F\left(x, \nabla u, \nabla^{2} u\right)\right)+(1-c)\left(\partial_{t} u+H(x, \nabla u)\right)=0 .
$$

Hence, $u$ satisfies the dynamic boundary condition in the viscosity sense.

1.5. Relation with other types of boundary conditions. Let us discuss relations between the dynamic boundary condition and other types of boundary conditions from the game-theoretic point of view.

We can modify the previous game rules for (DBP1) to find game interpretations for fully nonlinear parabolic equations with the Dirichlet or Neumann boundary condition. More precisely, by simply letting $\zeta_{\varepsilon} \equiv 0$ instead of taking (1.13), we can prove that the value function $u^{\varepsilon}$ converges to a viscosity solution of

$$
(\mathrm{DP} 1) \begin{cases}\partial_{t} u+F\left(x, \nabla u, \nabla^{2} u\right)=0 & \text { in } \Omega \times(0, \infty), \\ \partial_{t} u=0 & \text { on } \partial \Omega \times(0, \infty), \\ u(\cdot, 0)=u_{0} & \text { in } \bar{\Omega}\end{cases}
$$

with the boundary condition (1.30) in the viscosity sense. We remark that (1.30) seems to be the same as the Dirichlet condition

$$
u=u_{0} \quad \text { on } \partial \Omega \times(0, \infty)
$$


but it may give different behavior of the solutions; see [16, Section 5] and [28, Section 5.3]. In fact, solutions of (DP1) in general are not unique, as pointed out in [1, Example A.5].

In order to obtain the convergence of the game values in this case, we need to impose additional barrier assumptions on $F$ so as to guarantee that the Dirichlet boundary condition (1.31) is interpreted either in the strict sense. It turns out that under the barrier assumptions, both (1.30) and (1.31) are equivalent and we thus can show that $u^{\varepsilon}$ converges to the unique solution of the Cauchy-Dirichlet problem. Another option to show convergence of $u^{\varepsilon}$ is to understand (1.30) as the Dirichlet condition (1.31) in the state constraint sense $[46,47]$. This requires a different barrier assumption on $F$. More details will be given in Section 4.1.

On the other hand, in order to build a game-based approximation for the Neumann boundary problem

$$
(\mathrm{NP}) \begin{cases}\partial_{t} u+F\left(x, \nabla u, \nabla^{2} u\right)=0 & \text { in } \Omega \times(0, \infty), \\ \langle\nabla u, \nu\rangle=0 & \text { on } \partial \Omega \times(0, \infty), \\ u(\cdot, 0)=u_{0} & \text { in } \bar{\Omega},\end{cases}
$$

we take $A=\{0\}$ with $\gamma_{0}=\nu$, and let $f(x, 0)=g(x, 0)=0$ for all $x \in \partial \Omega$. Moreover, in the game rules, we fix arbitrarily $\tau \in(0,1)$ and replace $\zeta_{\varepsilon}$ in (1.14) and in (1.15) by $\varepsilon^{-\tau} \zeta_{\varepsilon}$. Heuristically speaking, this change of rules turns (1.29) into

$$
\partial_{t} u^{\varepsilon}+\eta_{\varepsilon}^{2} F\left(x_{\varepsilon}, \nabla u^{\varepsilon}, \nabla^{2} u^{\varepsilon}\right)+\varepsilon^{-\tau} \zeta_{\varepsilon}\left\langle\nabla u^{\varepsilon}, \nu(x)\right\rangle=o(1),
$$

whose limit as $\varepsilon \rightarrow 0$ leads to (1.32) due to the fact that $\varepsilon^{-\tau} \rightarrow \infty$. In Section 4.2 , we provide a game-theoretic approach to a general class of Neumann type boundary problems by generalizing the game setting above.

We emphasize that our modified game construction for the Neumann boundary condition here is different from that in $[30,14]$. In order to force the game states to stay in $\bar{\Omega}$, we adopt shrinking moves near the boundary while in the games proposed in $[30,14]$ either reflection about $\partial \Omega$ or projection onto $\partial \Omega$ is applied once the game position exits from $\bar{\Omega}$.

1.6. Fast evolution asymptotics and the limit elliptic problem. It is a natural question whether we can obtain a similar game interpretation for elliptic problems with dynamic boundary conditions. This is related to the following problem, which will be discussed in detail in Section 5. Let us consider the asymptotics for

$$
\begin{cases}\delta \partial_{t} u+F\left(x, \nabla u, \nabla^{2} u\right)=0 & \text { in } \Omega \times(0, \infty), \\ \partial_{t} u+H(x, \nabla u)=0 & \text { on } \partial \Omega \times(0, \infty), \\ u(\cdot, 0)=u_{0} & \text { in } \bar{\Omega},\end{cases}
$$

as the parameter $\delta>0$ tends to 0 . Here $F$ and $H$ are assumed to satisfy all assumptions as in Theorem 1.1. Suppose that a comparison principle holds for (DBP2) and let $u_{\delta}$ denote the unique solution of (DBP2) for any fixed $\delta>0$.

Under the linear dynamic boundary condition (1.4), asymptotic behavior for (DBP2) as $\delta \rightarrow 0$ is investigated in [1] for the eikonal equation and later in [25] for the heat equation. The main results of these papers state that, when $F(x, p, X)=|p|$ or $F(x, p, X)=-\operatorname{tr} X$, the solution $u_{\delta} \rightarrow U$ locally uniformly in $[0, \infty)$ as $\delta \rightarrow 0$, where $U$ is the unique solution 
of the dynamic boundary problem for the corresponding elliptic equation, that is,

$$
\text { (DBP3 } \begin{cases}F\left(x, \nabla u, \nabla^{2} u\right)=0 & \text { in } \Omega \times(0, \infty), \\ \partial_{t} u+H(x, \nabla u)=0 & \text { on } \partial \Omega \times(0, \infty), \\ u(\cdot, 0)=u_{\infty} & \text { in } \bar{\Omega} .\end{cases}
$$

We must emphasize that an initial layer emerges in the limit process; the initial value $u_{\infty}$ for the limit $U$ does not coincide with $u_{0}$ in general. In fact, by rescaling (DBP2) in the time variable, $u_{\infty}$ can be identified as the large-time profile of

$$
\begin{cases}\partial_{t} u+F\left(x, \nabla u, \nabla^{2} u\right)=0 & \text { in } \Omega \times(0, \infty), \\ \partial_{t} u=0 & \text { on } \partial \Omega \times(0, \infty), \\ u(\cdot, 0)=u_{0} & \text { in } \bar{\Omega} .\end{cases}
$$

Under appropriate assumptions, $u_{\infty}$ can thus be obtained by uniquely solving the stationary Dirichlet problem

$$
\text { (DP2) } \begin{cases}F\left(x, \nabla u, \nabla^{2} u\right)=0 & \text { in } \Omega, \\ u=u_{0} & \text { on } \partial \Omega .\end{cases}
$$

We are able to use the game method to show the asymptotic behavior for the general dynamic boundary problem (DBP2). Our result is actually slightly stronger. By adapting the game setting we can easily construct a game value $u^{\varepsilon, \delta}$ that converges to $u_{\delta}$ locally uniformly in $\bar{\Omega} \times[0, \infty)$ as $\varepsilon \rightarrow 0$. It turns out that $u^{\varepsilon, \delta} \rightarrow U$ locally uniformly as $(\varepsilon, \delta) \rightarrow 0$ as long as $\delta \geq \varepsilon^{\sigma}$ with $\sigma>0$ satisfying

$$
\begin{gathered}
\sigma<2-\max \left\{\alpha \sigma_{1}, \beta \sigma_{2}\right\}, \\
\sigma<1+\gamma-\beta \sigma_{2}, \\
\sigma<1+\gamma-\alpha\left(\sigma_{1}-1\right), \\
\sigma<2 \gamma-\beta\left(\sigma_{2}-1\right) .
\end{gathered}
$$

We refer the reader to Theorem 5.6 and Theorem 5.10 for a more precise statement and a detailed proof. This result can also be considered as a game-theoretic interpretation of dynamic boundary problems for elliptic equations like (DBP3).

We remark that $\sigma$ fulfilling (1.38)-(1.41) does exist thanks to (1.21)-(1.24). In particular, if $F$ is Lipschitz continuous, then $\sigma_{1}=\sigma_{2}=1$ and we only require that $0<\sigma<2 \gamma$. To heuristic understand such a condition, in this case we may still apply Taylor expansion to the associated dynamic programming equation and obtain a new version of (1.27) with $F$ replaced by $\delta^{-1} F$. In order to make the entire argument work again, we thus need to make sure that, in the term with $\varepsilon^{2} \eta_{\varepsilon}^{2}$ on the left hand side, the range of $|v|^{2}$ is wider than that of $\delta^{-1}$, which demands at least formally that $\sigma<2 \gamma$.

It is worth pointing out that a comparison principle for (DBP3) is needed in our rigorous proof of the convergence of $u^{\varepsilon, \delta}$. Since it is not available explicitly in the literature, for the sake of completeness, we provide it in Appendix A with a detailed and technical proof, following the idea in $[7,8]$.

Organization of the paper. In Section 2 we give a review on preliminaries of the dynamic boundary problem (DBP1) including the definition of viscosity solutions and comparison results. In Section 3, we give a rigorous proof of Theorem 1.1. Section 4 is devoted to discussions on the relations with the Dirichlet and Neumann boundary problems from the game-theoretic point of view. We study the asymptotic behavior for 
(DBP2) and a game interpretation of (DBP3) in Section 5. A comparison principle for (DBP3) is presented in Appendix A for the sake of completeness.

Acknowledgments. The work of the first author was supported by JSPS Grant-in-Aid for Young Scientists, No. 16K17621 and by the Sumitomo Foundation through Grant for Basic Science Research Projects No. 150973 and Inamori Foundation through Research Grants. The work of the second author was supported by JSPS Grant-in-Aid for Young Scientists, No. 16K17635 and by the Grant from Central Research Institute of Fukuoka University, No. 177102.

\section{PREliminaries on DYNAMiC BOUNDARY PROBlems}

For the reader's convenience, in this section we briefly review the definition of viscosity solutions and known results on well-posedness of (DBP1), especially the comparison principle. Let us start with a definition of viscosity solutions of (DBP1).

2.1. Definition of viscosity solutions. In the sequel, we denote by $\operatorname{USC}(\bar{\Omega} \times[0, \infty))$ (resp., $\operatorname{LSC}(\bar{\Omega} \times[0, \infty))$ ) the set of all locally bounded upper semicontinuous (resp., lower semicontinuous) functions in $\bar{\Omega} \times[0, \infty)$.

Definition 2.1. A function $u \in U S C(\bar{\Omega} \times[0, \infty))$ (resp., $u \in L S C(\bar{\Omega} \times[0, \infty))$ ) is said to be a subsolution (resp., supersolution) of (1.1)-(1.2) if whenever there exist a function $\varphi \in C^{2}(\bar{\Omega} \times[0, \infty))$ and $\left(x_{0}, t_{0}\right) \in \bar{\Omega} \times(0, \infty)$ such that $u-\varphi$ attains a strict maximum (resp., minimum) in $\bar{\Omega} \times(0, \infty)$ at $\left(x_{0}, t_{0}\right)$, then the following inequalities hold:

- If $x_{0} \in \Omega$, then we have

$$
\begin{aligned}
& \partial_{t} \varphi\left(x_{0}, t_{0}\right)+F\left(x_{0}, \nabla \varphi\left(x_{0}, t_{0}\right), \nabla^{2} \varphi\left(x_{0}, t_{0}\right)\right) \leq 0 \\
& \left.\left(\operatorname{resp} ., \partial_{t} \varphi\left(x_{0}, t_{0}\right)+F\left(x_{0}, \nabla \varphi\left(x_{0}, t_{0}\right), \nabla^{2} \varphi\left(x_{0}, t_{0}\right)\right) \geq 0\right)\right) .
\end{aligned}
$$

- If $x_{0} \in \partial \Omega$, then we have

$$
\begin{aligned}
& \partial_{t} \varphi\left(x_{0}, t_{0}\right)+\min \left\{F\left(x_{0}, \nabla \varphi\left(x_{0}, t_{0}\right), \nabla^{2} \varphi\left(x_{0}, t_{0}\right)\right), H\left(x_{0}, \nabla \varphi\left(x_{0}, t_{0}\right)\right)\right\} \leq 0 \\
& \left(\operatorname{resp} ., \partial_{t} \varphi\left(x_{0}, t_{0}\right)+\max \left\{F\left(x_{0}, \nabla \varphi\left(x_{0}, t_{0}\right), \nabla^{2} \varphi\left(x_{0}, t_{0}\right)\right), H\left(x_{0}, \nabla \varphi\left(x_{0}, t_{0}\right)\right)\right\} \geq 0\right) .
\end{aligned}
$$

A continuous function on $\bar{\Omega} \times[0, \infty)$ is called a solution of $(1.1)-(1.2)$ if it is both a subsolution and a supersolution.

Moreover, a function $u \in U S C(\bar{\Omega} \times[0, \infty)$ ) (resp., $u \in L S C(\bar{\Omega} \times[0, \infty)))$ is said to be a subsolution (resp., supersolution) of (DBP1) if it is a subsolution (supersolution) of $(1.1)-(1.2)$ and satisfies $u(\cdot, 0) \leq u_{0}$ (resp., $\left.u(\cdot, 0) \geq u_{0}\right)$ in $\bar{\Omega}$. A continuous function on $\bar{\Omega} \times[0, \infty)$ is a solution of (DBP1) if it is both a subsolution and a supersolution of (DBP1).

Remark 2.2. For a stationary equation with a dynamic boundary condition such as (DBP3), we can define its viscosity solutions in the same manner. 
2.2. Comparison results. It is known that a comparison principle holds for (DBP1) if $\Omega$ is a $C^{2,1}$ bounded domain, $H$ fulfills (1.11) and $F$ satisfies appropriate regularity assumptions. A typical regularity assumption on $F$ is as follows.

(F3) $F$ is a Lipschitz (degenerate) elliptic operator in the sense that there exists $L_{F}>0$ such that

$$
\begin{aligned}
& \left|F(x, p, X)-F\left(x^{\prime}, p^{\prime}, X^{\prime}\right)\right| \\
& \leq L_{F}\left(\left(1+|p|+\left|p^{\prime}\right|\right)\left|x-x^{\prime}\right|+\left|p-p^{\prime}\right|+\left|X-X^{\prime}\right|\right)
\end{aligned}
$$

for all $x, x^{\prime} \in \bar{\Omega}, p, p^{\prime} \in \mathbb{R}^{n}, X, X^{\prime} \in \mathbb{S}^{n}$, and

$$
F(x, p, X) \leq F\left(x, p, X^{\prime}\right)
$$

for any $x \in \bar{\Omega}, p \in \mathbb{R}^{n}$ and $X, X^{\prime} \in \mathbb{S}^{n}$ satisfying $X \geq X^{\prime}$.

Theorem 2.3 (Comparison theorem for (DBP1) [8, Theorem 3.2]). Assume that $\Omega \subset \mathbb{R}^{n}$ is a bounded $C^{2,1}$ domain. Assume that (F3), (1.10) and (1.11) hold. Let $u$ be a bounded subsolution of (1.1)-(1.2) and $v$ be a bounded supersolution of $(1.1)-(1.2)$. If $u(\cdot, 0) \leq$ $v(\cdot, 0)$ on $\bar{\Omega}$, then $u \leq v$ in $\bar{\Omega} \times[0, \infty)$.

Remark 2.4. The boundedness assumption on $u$ and $v$ is a technical assumption. Indeed, one can prove the comparison result in $\bar{\Omega} \times[0, T)$ for an arbitrarily $T>0$ if $u$ and $v$ are only locally bounded in $\bar{\Omega} \times[0, \infty)$; see the proof of an elliptic version (Theorem 2.5) below. We will use this comparison theorem below without assuming the boundedness of $u$ and $v$.

Without losing the comparison result, the assumption (F3) can be weakened; see [8, (H51)]. Since our main purpose is to give game-theoretic existence results, for our convenience in the game construction in Section 3 and Section 4, we will mainly use (F1)-(F2) and assume the comparison principle always holds, rather than assuming (F3) as well as $C^{2,1}$ regularity of $\Omega$.

In Section 5, since we will also discuss elliptic problems with dynamic boundary conditions, we need a comparison theorem as below to guarantee uniqueness of solutions. Besides (F3) and $C^{2,1}$ regularity of $\Omega$, we further impose the following assumption on the operator $F$ as well as the function $g$ given in the boundary Hamiltonian (1.8):

(F4) There are sequences $\lambda_{j}, c_{j}>0(j=1,2, \ldots)$ with

$$
\lambda_{j} \rightarrow 1, \quad c_{j} \rightarrow 0 \quad \text { as } j \rightarrow \infty
$$

such that

$$
F\left(x, \lambda_{j} p, \lambda_{j} X\right) \geq \lambda_{j} F(x, p, X)+c_{j}
$$

for all $j \geq 1, p \in \mathbb{R}^{n}$ and $X \in \mathbb{S}^{n}$ and

$$
\min _{(x, a) \in \bar{\Omega} \times A}\left(1-\lambda_{j}\right) g(x, a) \geq 0 .
$$

Theorem 2.5 (Comparison principle for elliptic equations with dynamic boundary conditions). Assume that $\Omega \subset \mathbb{R}^{n}$ is a bounded $C^{2,1}$ domain. Assume that (F3), (F4), (1.10) and (1.11) hold. Suppose that $u \in U S C(\bar{\Omega} \times[0, \infty))$ and $v \in L S C(\bar{\Omega} \times[0, \infty))$ are respectively a subsolution and a supersolution of (1.34) with dynamic boundary condition (1.2). If $u \leq v$ on $\bar{\Omega} \times\{0\}$, then $u \leq v$ in $\bar{\Omega} \times[0, \infty)$. 
The assumption (F4) amounts to saying that one can convert a supersolution (resp., subsolution) of (1.34) with (1.2) to a strict supersolution (resp., subsolution) in the interior by constant multiple, which plays an important role of proving the comparison principle. See [32] for a similar idea applied to the Hamilton-Jacobi equation.

As a typical example, if $\gamma_{a}=\nu, f(x, a)=0$, we see that (F4) is satisfied when $F(x, p, X)=|p|-b_{1}$, and $g(x, a)=b_{2}$ for all $x \in \bar{\Omega}, p \in \mathbb{R}^{n}$ and $X \in \mathbb{S}^{n}$ with $b_{1}>0$ and $b_{2} \leq 0$, i.e., the dynamic boundary problem reads

$$
\begin{cases}|\nabla u|=b_{1} & \text { in } \Omega \times(0, \infty), \\ \partial_{t} u+\langle\nabla u, \nu\rangle+b_{2}=0 & \text { on } \partial \Omega \times(0, \infty), \\ u(\cdot, 0)=u_{0} & \text { in } \bar{\Omega} .\end{cases}
$$

The assumption (F4) is also fulfilled in the same setting when $F(x, p, X)=-|p|-b_{1}$, $g(x, a)=b_{2}$ but with $b_{1}<0$ and $b_{2} \geq 0$.

As mentioned in Remark 2.4, here we also do not assume global boundedness but local boundedness on $u$ and $v$ in $\bar{\Omega} \times[0, \infty)$. A proof of Theorem 2.5 will be presented in detail in Section 5.2 and Appendix A.

\section{Convergence of game values}

This section is devoted to a rigorous proof of Theorem 1.1.

3.1. The rigorous proof. Under the conditions (1.20)-(1.24), let us define $S^{\varepsilon}: C(\bar{\Omega}) \times$ $\bar{\Omega} \times[0, \infty) \rightarrow \mathbb{R}$ to be

$$
S^{\varepsilon}[w](x)=\min _{p, X} \max _{a, v}\{w(x+h)-l\}
$$

for $w \in C(\bar{\Omega})$, where $h$ and $l$ are respectively given by (1.18) and (1.19). It is clear that $S^{\varepsilon}[w+C](x)=S^{\varepsilon}[w](x)+C$ for any constant $C \in \mathbb{R}, w \in C(\bar{\Omega})$ and $x \in \bar{\Omega}$. Moreover, it is not difficult to see that $S^{\varepsilon}$ is monotone in $w$, i.e.,

$$
S^{\varepsilon}\left[w_{1}\right](x) \geq S^{\varepsilon}\left[w_{2}\right](x)
$$

for any $x \in \bar{\Omega}$ and $w_{1}, w_{2} \in C(\bar{\Omega})$ with $w_{1} \geq w_{2}$ in $\bar{\Omega}$.

The following lemma plays a key role in the proof of Theorem 1.1.

Lemma 3.1 (Consistency). Suppose that $\Omega \subset \mathbb{R}^{n}$ is a bounded $C^{2}$ domain. Assume that (H1), (H2), (F1), (F2), (B1) and (B2) hold. Let $H$ be given by (1.8). Assume (1.20)-(1.24) and let $S^{\varepsilon}$ be defined by (3.1). Then for any $\varphi \in C^{2}(\bar{\Omega})$ and $x \in \bar{\Omega}$,

$$
S^{\varepsilon}[\varphi](x)-\varphi(x)=-\varepsilon^{2} \eta_{\varepsilon}^{2}(x) F\left(x, \nabla \varphi(x), \nabla^{2} \varphi(x)\right)-\varepsilon^{2} \zeta_{\varepsilon}(x) H(x, \nabla \varphi(x))+o\left(\varepsilon^{2}\right),
$$

where the constant implied in the error term o $\left(\varepsilon^{2}\right)$ is independent of $x \in \bar{\Omega}$.

Proof. For any $\varphi \in C^{2}(\bar{\Omega})$, by Taylor expansion we have

$$
S^{\varepsilon}[\varphi](x)-\varphi(x)=\min _{p, X} \max _{a, v}\left\{\langle\nabla \varphi(x), h\rangle+\frac{1}{2}\left\langle\nabla^{2} \varphi(x) h, h\right\rangle-l\right\}+o\left(\varepsilon^{2}\right)
$$

where $h$ and $l$ are given as in (1.18) and (1.19). The condition (1.20) is applied to deduce that $|h|^{3}$ is of order $o\left(\varepsilon^{2}\right)$. We may divide the right hand side of (3.3) by setting

$$
\min _{p, X} \max _{a, v}\left\{\langle\nabla \varphi(x), h\rangle+\frac{1}{2}\left\langle\nabla^{2} \varphi(x) h, h\right\rangle-l\right\}=Q_{1}+Q_{2},
$$


where

$$
\begin{gathered}
Q_{1}:=\min _{p, X} \max _{v}\left\{\sqrt{2} \varepsilon \eta_{\varepsilon}(x)\langle\nabla \varphi(x)-p, v\rangle+\varepsilon^{2} \eta_{\varepsilon}^{2}(x)\left(\left\langle\left(\nabla^{2} \varphi(x)-X\right) v, v\right\rangle-F(x, p, X)\right)\right\} ; \\
Q_{2}:=\max _{a \in A}\left\{\varepsilon^{2} \zeta_{\varepsilon}(x)\left\langle\nabla \varphi(x), f(x, a)-\gamma_{a}(x)\right\rangle-\varepsilon^{2} \zeta_{\varepsilon}(x) g(x, a)\right\} .
\end{gathered}
$$

We next claim that

$$
Q_{1}=-\varepsilon^{2} \eta_{\varepsilon}^{2}(x) F\left(x, \nabla \varphi(x), \nabla^{2} \varphi(x)\right)+o\left(\varepsilon^{2}\right) .
$$

The proof of the claim is essentially the same as that of [35, Lemma 4.1], except that all of the terms of the second order now carry $\eta_{\varepsilon}^{2}(x)$. We give a detailed proof below for the reader's convenience.

In order to prove that " $\leq$ " holds, one only needs to take $p=\nabla \varphi(x)$ and $X=\nabla^{2} \varphi(x)$ in (3.3). It suffices to show " $\geq$ " holds.

Roughly speaking, the assumptions (1.22)-(1.24) imply that the first term on the right hand side of (3.4) is the leading one in terms of the order in $\varepsilon$, which forces $p$ to be taken near $\nabla \varphi(x)$.

More precisely, by (1.22) we can take $q \in \mathbb{R}$ satisfying

$$
0<q<\min \left\{1-\gamma-\beta, \gamma+1-\beta \sigma_{2}\right\}
$$

and discuss the following cases.

a) Suppose $|\nabla \varphi(x)-p| \leq \varepsilon^{q}$ and $\nabla^{2} \varphi(x) \leq X+\varepsilon^{\gamma} I$. Then we may take $v=0$ so that

$$
Q_{1} \geq-\varepsilon^{2} \eta_{\varepsilon}^{2}(x) \max _{p, X} F(x, p, X) \geq-\varepsilon^{2} \eta_{\varepsilon}^{2}(x) F\left(x, \nabla \varphi(x), \nabla^{2} \varphi(x)\right)-L \varepsilon^{2} \eta_{\varepsilon}^{2}(x)\left(\varepsilon^{q}+\varepsilon^{\gamma}\right),
$$

where $L=L_{F}(R)$ is the local Lipschitz constant of $F$ in $(\mathrm{F} 1)$ for $R=1+\|\varphi\|_{C^{2}(\bar{\Omega})}$.

b) Suppose $|\nabla \varphi(x)-p| \leq \varepsilon^{q}$ and $X+\varepsilon^{\gamma} I-\nabla^{2} \varphi(x)$ is not positive semi-definite. Denote by $\lambda$ the maximum positive eigenvalue of $\nabla^{2} \varphi(x)-X-\varepsilon^{\gamma} I$. Let $v$ be an eigenvector associated to the eigenvalue $\lambda$ with $|v|=\varepsilon^{-\gamma}$. Moreover, we may adjust the sign of $v$ such that

$$
\langle\nabla \varphi(x)-p, v\rangle \geq 0 \text {. }
$$

By the Lipschitz continuity (F1) of $F$ and the growth condition (F2) again, it follows that

$$
\begin{aligned}
Q_{1} & \geq-\varepsilon^{2} \eta_{\varepsilon}^{2}(x) F\left(x, \nabla \varphi(x), \nabla^{2} \varphi(x)-\lambda I\right)+\varepsilon^{2} \eta_{\varepsilon}^{2}(x)\left(\lambda \varepsilon^{-2 \gamma}+\varepsilon^{-\gamma}\right)-L \varepsilon^{2} \eta_{\varepsilon}^{2}(x)\left(\varepsilon^{q}+\varepsilon^{\gamma}\right) \\
& \geq-\varepsilon^{2} \eta_{\varepsilon}^{2}(x) F\left(x, \nabla \varphi(x), \nabla^{2} \varphi(x)\right)+\varepsilon^{2} \eta_{\varepsilon}^{2}(x)\left(\lambda \varepsilon^{-2 \gamma}-C \lambda^{\sigma_{2}}\right)+o\left(\varepsilon^{2}\right)
\end{aligned}
$$

for some $C>0$ large. Noticing that $\lambda \leq C\left(1+\varepsilon^{-\beta}\right)$, we have

$$
\lambda \varepsilon^{-2 \gamma}-C \lambda^{\sigma_{2}} \geq \lambda\left(\varepsilon^{-2 \gamma}-C \lambda^{\sigma_{2}-1}\right) \geq 0
$$

when $\varepsilon>0$ is small, since (1.24) holds. We therefore get the desired inequality in this case too.

c) Suppose that $|\nabla \varphi(x)-p|>\varepsilon^{q}$. Take $v$ parallel to $\nabla \varphi(x)-p$ with $|v|=\varepsilon^{-\gamma}$. Then we get the estimate by (F2),

$$
\begin{aligned}
Q_{1} & \geq \sqrt{2} \varepsilon^{1-\gamma} \eta_{\varepsilon}(x)|\nabla \varphi(x)-p|-C \eta_{\varepsilon}^{2}(x)\left(\varepsilon^{2-\beta-2 \gamma}+\varepsilon^{2-\beta \sigma_{2}}\right)-C \varepsilon^{2} \eta_{\varepsilon}^{2}(x)|p|^{\sigma_{1}} \\
& \geq \varepsilon^{1-\gamma} \eta_{\varepsilon}(x)|\nabla \varphi(x)-p|-C \varepsilon^{2} \eta_{\varepsilon}^{2}(x)|p|^{\sigma_{1}}
\end{aligned}
$$


for some $C>0$ when $\varepsilon>0$ is sufficiently small. The last inequality is due to (3.7) and the fact that $0 \leq \eta_{\varepsilon} \leq 1$. If $|p| \leq 2|\nabla \varphi(x)|$, then for $\varepsilon>0$ small we have

$$
\begin{aligned}
\varepsilon^{1-\gamma} \eta_{\varepsilon}(x)|\nabla \varphi(x)-p|-C \varepsilon^{2} \eta_{\varepsilon}^{2}(x)|p|^{\sigma_{1}} & \geq \varepsilon^{1-\gamma} \eta_{\varepsilon}(x)|\nabla \varphi(x)-p|-O\left(\varepsilon^{2}\right) \\
& \geq \varepsilon^{1-\gamma+q} \eta_{\varepsilon}(x)-O\left(\varepsilon^{2}\right) .
\end{aligned}
$$

Suppose, on the other hand, that $|p|>2|\nabla \varphi(x)|$, which implies that $|p| \geq 2 \varepsilon^{q} / 3$, then

$$
\varepsilon^{1-\gamma} \eta_{\varepsilon}|\nabla \varphi(x)-p|-C \varepsilon^{2} \eta_{\varepsilon}^{2}|p|^{\sigma_{1}} \geq \frac{1}{2} \varepsilon^{1-\gamma} \eta_{\varepsilon}|p|-C \varepsilon^{2} \eta_{\varepsilon}^{2}|p|^{\sigma_{1}}
$$

Note that

$$
\begin{aligned}
\frac{1}{2} \varepsilon^{1-\gamma} \eta_{\varepsilon}(x)|p|-C \varepsilon^{2} \eta_{\varepsilon}^{2}|p|^{\sigma_{1}} & \geq \varepsilon^{1-\gamma} \eta_{\varepsilon}^{2}(x)|p|\left(\frac{1}{2}-C \varepsilon^{1+\gamma}|p|^{\sigma_{1}-1}\right) \\
& \geq \varepsilon^{1-\gamma} \eta_{\varepsilon}^{2}(x)|p|\left(\frac{1}{2}-C \varepsilon^{1+\gamma-\alpha\left(\sigma_{1}-1\right)}\right) .
\end{aligned}
$$

It follows from (1.23) that

$$
\varepsilon^{1-\gamma} \eta_{\varepsilon}(x)|p|-C \varepsilon^{2} \eta_{\varepsilon}^{2}(x)|p|^{\sigma_{1}} \geq \frac{1}{4} \varepsilon^{1-\gamma} \eta_{\varepsilon}^{2}(x)|p| \geq \frac{1}{6} \varepsilon^{1-\gamma+q} \eta_{\varepsilon}^{2}(x)
$$

when $\varepsilon>0$ is small. In either case, we obtain

$$
Q_{1} \geq-\varepsilon^{2} \eta_{\varepsilon}^{2}(x) F\left(x, \nabla \varphi(x), \nabla^{2} \varphi(x)\right) .
$$

We thus complete the proof of the claim.

Moreover, it is clear that

$$
Q_{2}=\max _{a \in A}\left\{\varepsilon^{2} \zeta_{\varepsilon}(x)\left\langle\nabla \varphi(x), f(x, a)-\gamma_{a}(x)\right\rangle-\varepsilon^{2} \zeta_{\varepsilon}(x) g(x, a)\right\}=-\varepsilon^{2} \zeta_{\varepsilon}(x) H(x, \nabla \varphi(x)) \text {. }
$$

We conclude the proof of the lemma by plugging (3.6) and (3.8) together with (3.4) and (3.5) into (3.3).

Proposition 3.2 (Characterization of relaxed limits as sub- and supersolutions). Suppose that $\Omega \subset \mathbb{R}^{n}$ is a bounded $C^{2}$ domain. Assume (H1), (H2), (F1), (F2), (B1) and (B2) hold. Let $H$ be given by (1.8). Assume (1.20)-(1.24). Assume that $u_{0}$ is Lipschitz in $\bar{\Omega}$. Let $u^{\varepsilon}$ be the value function defined as in (1.16) for any $\varepsilon>0$ small. Then $\bar{u}$ and $\underline{u}$ given in (1.25) are respectively a subsolution and a supersolution of (1.1)-(1.2).

Proof. Let us first show that $\bar{u}$ is a subsolution of (1.1)-(1.2). Suppose that there exist $\left(x_{0}, t_{0}\right) \in \bar{\Omega} \times(0, \infty)$ and $\phi \in C^{2}(\bar{\Omega} \times[0, \infty))$ such that $\bar{u}-\phi$ attains a unique maximum over $\bar{\Omega} \times[0, \infty)$ at $\left(x_{0}, t_{0}\right)$. The proof is precisely the same as that of [35, Proposition 3.3] if $x_{0} \in \Omega$. We therefore only consider the boundary case, that is, $x_{0} \in \partial \Omega$.

By the definition of $\bar{u}$, we may find $r>0$ and a sequence $\left(x_{\varepsilon}, t_{\varepsilon}\right) \in \bar{\Omega} \times(0, \infty)$ still indexed by $\varepsilon$ such that $\left(x_{\varepsilon}, t_{\varepsilon}\right) \rightarrow\left(x_{0}, t_{0}\right)$ as $\varepsilon \rightarrow 0$ and

$$
\left(u^{\varepsilon}-\phi\right)\left(x_{\varepsilon}, t_{\varepsilon}\right) \geq \sup _{(x, t) \in B_{r}\left(x_{0}, t_{0}\right)}\left(u^{\varepsilon}-\phi\right)(x, t)-\varepsilon^{3} .
$$

By (1.17), we get

$$
\phi\left(x_{\varepsilon}, t_{\varepsilon}\right) \leq S^{\varepsilon}\left[\varphi_{\varepsilon}\right]\left(x_{\varepsilon}\right)+\varepsilon^{3},
$$

where $S^{\varepsilon}$ is defined in (3.1) and

$$
\varphi_{\varepsilon}:=\phi\left(\cdot, t_{\varepsilon}-\varepsilon^{2}\right) \in C^{2}(\bar{\Omega}) .
$$


It follows from Lemma 3.1 with $\varphi=\varphi_{\varepsilon}$ that

$\phi\left(x_{\varepsilon}, t_{\varepsilon}\right) \leq \varphi_{\varepsilon}\left(x_{\varepsilon}\right)-\varepsilon^{2} \eta_{\varepsilon}^{2}\left(x_{\varepsilon}\right) F\left(x_{\varepsilon}, \nabla \varphi_{\varepsilon}\left(x_{\varepsilon}\right), \nabla^{2} \varphi_{\varepsilon}\left(x_{\varepsilon}\right)\right)-\varepsilon^{2} \zeta_{\varepsilon}\left(x_{\varepsilon}\right) H\left(x_{\varepsilon}, \nabla \varphi_{\varepsilon}\left(x_{\varepsilon}\right)\right)+o\left(\varepsilon^{2}\right)$,

which implies that

$$
\varepsilon^{2} \partial_{t} \phi\left(x_{\varepsilon}, t_{\varepsilon}\right) \leq-\varepsilon^{2} \eta_{\varepsilon}^{2}\left(x_{\varepsilon}\right) F\left(x_{\varepsilon}, \nabla \varphi_{\varepsilon}\left(x_{\varepsilon}\right), \nabla^{2} \varphi_{\varepsilon}\left(x_{\varepsilon}\right)\right)-\varepsilon^{2} \zeta_{\varepsilon}\left(x_{\varepsilon}\right) H\left(x_{\varepsilon}, \nabla \varphi_{\varepsilon}\left(x_{\varepsilon}\right)\right)+o\left(\varepsilon^{2}\right) .
$$

By taking a further subsequence, we may assume that $\eta_{\varepsilon}^{2}\left(x_{\varepsilon}\right) \rightarrow c$ as $\varepsilon \rightarrow 0$ for some $c \in[0,1]$. Then dividing both sides of $(3.9)$ by $\varepsilon^{2}$ and letting $\varepsilon \rightarrow 0$ yield

$$
\partial_{t} \phi\left(x_{0}, t_{0}\right)+c F\left(x_{0}, \nabla \phi\left(x_{0}, t_{0}\right), \nabla^{2} \phi\left(x_{0}, t_{0}\right)\right)+(1-c) H\left(x_{0}, \nabla \phi\left(x_{0}, t_{0}\right)\right) \leq 0,
$$

which in turn implies that either of the following inequalities holds:

$$
\begin{gathered}
\partial_{t} \phi\left(x_{0}, t_{0}\right)+F\left(x_{0}, \nabla \phi\left(x_{0}, t_{0}\right), \nabla^{2} \phi\left(x_{0}, t_{0}\right)\right) \leq 0, \\
\partial_{t} \phi\left(x_{0}, t_{0}\right)+H\left(x_{0}, \nabla \phi\left(x_{0}, t_{0}\right)\right) \leq 0 .
\end{gathered}
$$

The proof for $\bar{u}$ is thus complete. One can similarly show that $\underline{u}$ is a supersolution.

We also need to establish comparison between the initial values of $\bar{u}$ and $\underline{u}$.

Proposition 3.3 (Initial comparison). Suppose that $\Omega \subset \mathbb{R}^{n}$ is a bounded $C^{2}$ domain. Assume that (H1), (H2), (F1), (F2), (B1) and (B2) hold. Assume that (1.20)-(1.24) hold. Let $H$ be given by (1.8). Assume that $u_{0}$ is Lipschitz in $\bar{\Omega}$. Let $u^{\varepsilon}$ be the value function defined as in (1.16) for any $\varepsilon>0$ small. Let $\bar{u}$ and $\underline{u}$ be the relaxed limits given in (1.25). Then

$$
\bar{u}(\cdot, 0)=u_{0}=\underline{u}(\cdot, 0) \quad \text { in } \bar{\Omega} .
$$

Proof. Let us prove that $\bar{u}\left(x_{0}, 0\right) \leq u_{0}\left(x_{0}\right)$ for any fixed $x_{0} \in \bar{\Omega}$. Since $u_{0}$ is Lipschitz continuous, for any fixed $\tau>0$ there exists a constant $C_{\tau}>0$ such that

$$
u_{0}(x) \leq W_{\tau}(x):=u_{0}\left(x_{0}\right)+\tau+C_{\tau}\left|x-x_{0}\right|^{2} \quad \text { for all } x \in \bar{\Omega} .
$$

By Lemma 3.1, we have

$$
\begin{aligned}
S^{\varepsilon}\left[W_{\tau}\right](x)-W_{\tau}(x)=-\varepsilon^{2} \eta_{\varepsilon}^{2}(x) F(x, & \left.\nabla W_{\tau}(x), \nabla^{2} W_{\tau}(x)\right) \\
& -\varepsilon^{2} \zeta_{\varepsilon}(x) H\left(x, \nabla W_{\tau}(x)\right)+o\left(\varepsilon^{2}\right)
\end{aligned}
$$

for all $x \in \bar{\Omega}$. Here the constant implied in the error term is uniform for $x \in \bar{\Omega}$. We also choose a constant $K>0$ such that

$$
\left|F\left(x, \nabla W_{\tau}(x), \nabla^{2} W_{\tau}(x)\right)\right|+\left|H\left(x, \nabla W_{\tau}(x)\right)\right| \leq K
$$

for all $x \in \bar{\Omega}$. It follows that for each $x \in \bar{\Omega}$,

$$
S^{\varepsilon}\left[W_{\tau}\right](x)-W_{\tau}(x) \leq K \varepsilon^{2}+o\left(\varepsilon^{2}\right) .
$$

Hence, by the definition and monotonicity of $S^{\varepsilon}$ we see that

$$
u^{\varepsilon}\left(x, \varepsilon^{2}\right)=S^{\varepsilon}\left[u_{0}\right](x) \leq S^{\varepsilon}\left[W_{\tau}\right](x) \leq W_{\tau}(x)+K \varepsilon^{2}+o\left(\varepsilon^{2}\right)
$$

for all $x \in \bar{\Omega}$.

Let $t>0$. After repeating this estimate $N$ times with $N=\left[t / \varepsilon^{2}\right]$, we obtain

$$
u^{\varepsilon}(x, t) \leq W_{\tau}(x)+K N \varepsilon^{2}+o\left(N \varepsilon^{2}\right) \leq W_{\tau}(x)+K t+o(1)
$$

for all $x \in \bar{\Omega}$. Taking the relaxed half limit $\varepsilon \rightarrow 0$ implies

$$
\bar{u}(x, t) \leq W_{\tau}(x)+K t
$$


for all $x \in \bar{\Omega}$. This in particular gives

$$
\bar{u}\left(x_{0}, 0\right) \leq W_{\tau}\left(x_{0}\right)=u_{0}\left(x_{0}\right)+\tau,
$$

and therefore sending $\tau \rightarrow 0$ yields that $\bar{u}\left(x_{0}, 0\right) \leq u_{0}\left(x_{0}\right)$.

A symmetric proof gives $\underline{u}\left(x_{0}, 0\right) \geq u_{0}\left(x_{0}\right)$. We thus conclude the proof, since by definition it is clear that $\bar{u}\left(x_{0}, 0\right) \geq \underline{u}\left(x_{0}, 0\right)$.

The statements in Theorem 1.1 are immediate consequences of Proposition 3.2 and Proposition 3.3.

Remark 3.4. Replacing (F1) and (F2) by a stronger assumption (F3), we can obtain the convergence $u^{\varepsilon} \rightarrow u$ due to the comparison principle in Theorem 2.3. Under the assumption (F3), the conditions (1.20)-(1.24) on $\alpha, \beta, \gamma$ become simpler, since $\sigma_{1}$ and $\sigma_{2}$ can be chosen to be 1 . In fact, in this case we only require that

$$
\gamma<\frac{1}{3}, \quad \alpha+\gamma<1, \quad \beta+\gamma<1
$$

3.2. Variants of the game. A straightforward observation, based on our formal proof, is that if we switch the goals of both players by taking $\max _{p, X} \min _{a, v}$ instead of $\min _{p, X} \max _{a, v}$ in the game rules, then the game value is turned into an approximation for (DBP1) with

$$
H(x, p)=\max _{a \in A}\left\{\left\langle p, \gamma_{a}(x)-f(x, a)\right\rangle+g(x, a)\right\}
$$

instead of (1.8).

Moreover, we may generate an even more general Hamiltonian $H$ of Isaacs type via our games by including one more controller; more precisely, given another compact metric space $B$, suppose that the oblique normal and the functions $f, g$ now depend also on $b \in B$. Denote the oblique normal by $\gamma_{a, b}$ and assume

$$
\inf _{\substack{x \in \partial \Omega \\ a \in A, b \in B}}\left\langle\gamma_{a, b}(x), \nu(x)\right\rangle>0 .
$$

If $H: \bar{\Omega} \times \mathbb{R}^{n} \rightarrow \mathbb{R}$ can be expressed as

$$
H(x, p)=\max _{b \in B} \min _{a \in A}\left\{\left\langle p, \gamma_{a, b}(x)-f(x, a, b)\right\rangle+g(x, a, b)\right\},
$$

we can build a new game by following the same rules but letting Player I also choose $b \in B$ at each step besides the controls $p$ and $X$. The value function is now defined by

$$
u^{\varepsilon}(x, t)=\min _{b_{1}, p_{1}, X_{1}} \max _{a_{1}, v_{1}} \min _{b_{2}, p_{2}, X_{2}} \max _{a_{2}, v_{2}} \ldots \min _{b_{N}, p_{N}, X_{N}} \max _{a_{N}, v_{N}} J^{\varepsilon}(x, t) .
$$

Since this change only affects the Taylor expansion of $Q_{2}$ near the boundary, the limit $u$ still satisfies (1.1) in the interior. Meanwhile, the boundary condition with this new Hamiltonian $H$ in (3.13) can be derived analogously. We choose to keep our exhibition simple in this work and refer the reader to [21] for technical details on this possible generalization.

\section{Relation With other Boundary pRoblems}

Based on the method in the previous section, we can obtain game interpretations for the Dirichlet and Neumann boundary problems by slightly modifying the rules. 
4.1. Reduction to the Dirichlet problem. If we keep the definition of $\eta_{\varepsilon}$ in (1.12) but letting $\zeta_{\varepsilon} \equiv 0$ instead of requiring (1.13), we can follow the whole argument in the previous section to derive the corresponding PDE. It is easily observed that the only difference lies at the expansion (1.29) on the boundary, which in the current case reads

$$
\partial_{t} u^{\varepsilon}\left(x_{\varepsilon}, t_{\varepsilon}\right)+\eta_{\varepsilon}^{2}\left(x_{\varepsilon}\right) F\left(x_{\varepsilon}, \nabla u^{\varepsilon}\left(x_{\varepsilon}, t_{\varepsilon}\right), \nabla^{2} u^{\varepsilon}\left(x_{\varepsilon}, t_{\varepsilon}\right)\right)=o(1),
$$

Passing to the limit yields that

$$
\partial_{t} u(x, t)+c F\left(x, \nabla u, \nabla^{2} u\right)=c\left(\partial_{t} u+F\left(x, \nabla u, \nabla^{2} u\right)\right)+(1-c) \partial_{t} u=0
$$

holds for some $c \in[0,1]$. This suggests that $u$ satisfies the boundary condition $\partial_{t} u=0$ in the viscosity sense; in practice, we should verify this by using inequalities rather than equalities. Hence, the modified game should give rise to (DP1). We can still characterize the relaxed limits $\bar{u}$ and $\underline{u}$ as a sub- and a supersolution of (DP1) respectively.

Corollary 4.1 (Degenerate dynamic boundary problems). Assume that $\Omega \subset \mathbb{R}^{n}$ is a bounded $C^{2}$ domain. Assume that (F1) and (F2) hold. Assume that $u_{0}$ is Lipschitz in $\bar{\Omega}$. For any $\varepsilon>0$ small, let $u^{\varepsilon}$ be the value function, defined as in (1.16), for the game with $\zeta_{\varepsilon} \equiv 0$. Then $\bar{u}$ and $\underline{u}$ given in (1.25) are respectively a subsolution and a supersolution of (DP1).

Remark 4.2. It is also possible to have a game for (DP1) by still having (1.13) and substituting $\zeta_{\varepsilon}$ with $\varepsilon^{\tau} \zeta_{\varepsilon}$ for any fixed $\tau \in(0,1)$; the expansion (1.29) on the boundary implies

$$
\partial_{t} u^{\varepsilon}+\eta_{\varepsilon}^{2} F\left(x_{\varepsilon}, \nabla u^{\varepsilon}, \nabla^{2} u^{\varepsilon}\right)+\varepsilon^{\tau} \zeta_{\varepsilon} H\left(x_{\varepsilon}, \nabla u^{\varepsilon}\right)=o(1) .
$$

It is then straightforward to get (1.30) in the viscosity sense. This method seems more complicated, but introducing such an additional parameter $\tau>0$ enables us to compare with the games for the classical Neumann condition in Section 4.2.

Concerning the initial data, the result in Lemma 3.3 still holds even if we take $\zeta_{\varepsilon}=0$. However, we are not able to prove the convergence of $u^{\varepsilon}$, since the boundary Hamiltonian does not satisfy (1.11) and comparison results are not known in general. In fact, viscosity solutions of (DP1) are in general not unique, as shown in [1, Example A.5].

We next discuss two distinct ways to understand the boundary condition $\partial_{t} u=0$, depending on different assumptions on $F$.

4.1.1. Strict Dirichlet boundary condition. The problem (DP1) looks equivalent, at least formally from the game perspective, to the following Cauchy-Dirichlet problem

$$
\text { (DP3) } \begin{cases}\partial_{t} u+F\left(x, \nabla u, \nabla^{2} u\right)=0 & \text { in } \Omega \times(0, \infty), \\ u=u_{0} & \text { on } \bar{\Omega} \times\{0\} \text { and } \partial \Omega \times(0, \infty) .\end{cases}
$$

In fact, due to the choice $\zeta_{\varepsilon} \equiv 0$, the interior game dynamics gradually vanishes when approaching the boundary; such kind of method to generate the Dirichlet boundary condition is employed in [5] in the Tug-of-war game for $\infty$-Laplacian.

A rigorous analysis for the relation between (DP1) and (DP3) (either a proof of equivalence or counterexamples to indicate their difference) would be interesting. We below show the equivalence in special cases with additional assumptions.

The game above precisely characterizes the classical Dirichlet problem (DP3) provided that boundary barriers can be constructed from both above and below. In order to guarantee the convergence of game values, we need a comparison principle in the following form: 
(CP1) Let $u \in U S C(\bar{\Omega} \times[0, \infty))$ and $v \in L S C(\bar{\Omega} \times[0, \infty))$ be respectively a subsolution and a supersolution of (1.1). If $u(x, t) \leq v(x, t)$ for any $(x, t) \in \bar{\Omega} \times\{0\}$ and $(x, t) \in \partial \Omega \times(0, \infty)$, then $u \leq v$ in $\bar{\Omega} \times[0, \infty)$.

Besides, we also assume the existence of barriers at any boundary points:

(F5) For any $x_{0} \in \partial \Omega$ and $\tau>0$, there exists $\psi_{\tau}^{ \pm} \in C^{2}(\bar{\Omega})$ such that

$$
\begin{gathered}
\psi_{\tau}^{+}\left(x_{0}\right)-\tau \leq u_{0}\left(x_{0}\right) \leq \psi_{\tau}^{-}\left(x_{0}\right)+\tau, \\
\psi_{\tau}^{-} \leq u_{0} \leq \psi_{\tau}^{+} \quad \text { in } \bar{\Omega}, \quad \text { and } \\
F\left(x, \nabla \psi_{\tau}^{+}(x), \nabla^{2} \psi_{\tau}^{+}(x)\right) \geq 0, \quad F\left(x, \nabla \psi_{\tau}^{-}(x), \nabla^{2} \psi_{\tau}^{-}(x)\right) \leq 0 \quad \text { for all } x \in \Omega .
\end{gathered}
$$

Theorem 4.3 (Classical Dirichlet boundary problems). Assume that $\Omega \subset \mathbb{R}^{n}$ is a bounded $C^{2}$ domain. Assume that (F1), (F2) and (F5) hold. Assume (1.20)-(1.24). Assume that $u_{0}$ is Lipschitz in $\bar{\Omega}$. Let $u^{\varepsilon}$ be the value function, defined as in (1.16), of the game for (DP1) with $\zeta_{\varepsilon} \equiv 0$. Then $\bar{u}$ and $\underline{u}$ given in (1.25) are respectively a subsolution and $a$ supersolution of (1.1) satisfying (3.10) and

$$
\bar{u}(\cdot, t)=u_{0}=\underline{u}(\cdot, t) \quad \text { on } \partial \Omega \text { for all } t \geq 0 .
$$

Moreover, if the comparison principle (CP1) holds, then $u^{\varepsilon} \rightarrow u$ locally uniformly in $\bar{\Omega} \times[0, \infty)$ as $\varepsilon \rightarrow 0$, where $u$ is the unique solution of (DP3).

Proof. Our results in the previous sections already show that $\bar{u}$ and $\underline{u}$ given in (1.25) are respectively a subsolution and a supersolution of (1.1) satisfying (3.10). It remains to prove (4.2). Let us fix $\tau>0, x_{0} \in \partial \Omega$ and take $\psi_{\tau}^{+}$as given in (F5). Since $S^{\varepsilon}$ is monotone and $u_{0} \leq \psi_{\tau}^{+}$in $\bar{\Omega}$, we have

$$
u^{\varepsilon}\left(x, \varepsilon^{2}\right)=S^{\varepsilon}\left[u_{0}\right](x) \leq S^{\varepsilon}\left[\psi_{\tau}^{+}\right](x) \text { in } \bar{\Omega} .
$$

By the consistency result in Lemma 3.1, it follows that

$$
u^{\varepsilon}\left(x, \varepsilon^{2}\right) \leq \psi_{\tau}^{+}(x)-\varepsilon^{2} \eta_{\varepsilon}^{2} F\left(\nabla \psi_{\tau}^{+}(x), \nabla^{2} \psi_{\tau}^{+}(x)\right)+o\left(\varepsilon^{2}\right)
$$

for all $x \in \bar{\Omega}$. In view of the assumption (4.1), we thus get

$$
u^{\varepsilon}\left(x, \varepsilon^{2}\right) \leq \psi_{\tau}^{+}(x)+o\left(\varepsilon^{2}\right)
$$

for all $x \in \bar{\Omega}$. Since $u^{\varepsilon}\left(x, \varepsilon^{2}\right)=u_{0}(x)$ for $x \in \partial \Omega$ by definition, we obtain (4.3) for all $x \in \bar{\Omega}$.

Iterating this argument yields

$$
u^{\varepsilon}(x, t) \leq \psi_{\tau}^{+}(x)+o(1)
$$

for all $x \in \bar{\Omega}$ and $t \geq 0$. Letting $\varepsilon \rightarrow 0$ and then $\tau \rightarrow 0$, we end up with

$$
\bar{u}\left(x_{0}, t\right) \leq u_{0}\left(x_{0}\right)
$$

for all $t \geq 0$. We may apply a symmetric argument to deduce that

$$
\underline{u}\left(x_{0}, t\right) \geq u_{0}\left(x_{0}\right)
$$

and the proof of (4.2) is complete.

Such boundary barriers are also indispensable when one attempts to use Perron's method to show existence of solutions to the classical Dirichlet problem (DP3). We refer to [13] for concrete examples on how to construct the barriers. 
4.1.2. State constraint boundary condition. On the other hand, on some occasions the Dirichlet condition are interpreted in the viscosity solution sense especially when one side of the boundary barriers is lost. This corresponds to the so-called state constraint boundary condition; consult $[46,47,10,33,6]$ etc. for introduction on this notion of solutions. We thus can characterize limits of game values as the state constraint solutions to (DP3) in place of viscosity solutions to (DP1).

We recall that $u \in L S C(\bar{\Omega})$ is said to be a state constraint supersolution of (1.1) if $u$ satisfies

$$
\partial_{t} u(x, t)+F\left(x, \nabla u(x, t), \nabla^{2} u(x, t)\right) \geq 0
$$

for all $(x, t) \in \bar{\Omega} \times(0, \infty)$ in the viscosity sense; a precise definition follows that in [33] for elliptic equations. A function $u \in C(\bar{\Omega} \times[0, \infty))$ is called a state constraint solution of (1.1) if it is both a subsolution and a state constraint supersolution of (1.1). It is called a state constraint solution of (DP3) if it also satisfies the initial condition (1.3).

In this case, the following comparison principle is needed:

(CP2) Let $u \in U S C(\bar{\Omega})$ be a subsolution of (1.1) and $v \in L S C(\bar{\Omega})$ be a state constraint supersolution of (1.1). If $u \leq v$ on $\bar{\Omega} \times\{0\}$, then $u \leq v$ in $\Omega \times[0, \infty)$.

Remark 4.4. We remark that in general it is challenging to prove (CP2). Even in the first order case, the uniform continuity of $u$ or $v$ is required in the proof [10]. Moreover, even if (CP2) holds, one cannot expect that $u \leq v$ on $\partial \Omega \times[0, \infty)$; a concrete example is given in Example 4.8.

Below we show the convergence of game values to state constraint solutions when one side of the barriers is lost. Formally speaking, if there exists no subsolutions of (DP2) below $u_{0}$, then the interior evolution somehow forces $\partial_{t} u<0$ in $\bar{\Omega} \times(0, \infty)$, which in turn requires the boundary condition to be (4.4) for $(x, t) \in \partial \Omega \times(0, \infty)$.

To give a more precise statement below, for any $\tau>0$, we set

$$
\Psi_{\tau}=\left\{\psi \in C^{2}(\bar{\Omega}): \psi \leq u_{0} \text { in } \bar{\Omega} \text { and }-\tau \leq \psi-u_{0} \leq 0 \text { on } \partial \Omega\right\} .
$$

Theorem 4.5 (State constraint boundary problems). Assume that $\Omega \subset \mathbb{R}^{n}$ is a bounded $C^{2}$ domain. Assume that (F1) and (F2) hold. Assume (1.20)-(1.24). Assume that $u_{0}$ is Lipschitz in $\bar{\Omega}$. Let $u^{\varepsilon}$ be the value function, defined as in (1.16), of the game for (DP1) with $\zeta_{\varepsilon} \equiv 0$. Assume in addition that there exists $\theta>0$ such that for any $\tau>0$ small and $\psi \in \Psi_{\tau}$ as in (4.5), there holds

$$
F\left(x, \nabla \psi(x), \nabla^{2} \psi(x)\right) \geq \theta \quad \text { for all } x \in \Omega .
$$

Let $\bar{u}$ and $\underline{u}$ be the relaxed limits given in (1.25). Then $\bar{u}$ is a subsolution of (1.1) and $\underline{u}$ is a state constraint supersolution of (1.1) with (3.10). Moreover, if the comparison principle (CP2) for the state constraint problem holds, then $u^{\varepsilon} \rightarrow u$ locally uniformly in $\Omega \times[0, \infty)$ as $\varepsilon \rightarrow 0$, where $u$ is the unique state constraint solution of (DP3).

Remark 4.6. The assumption involving (4.6) is quite restrictive for an elliptic operator $F$. In fact, it hardly holds when $F$ is uniformly elliptic. However, it can be satisfied when $F$ is highly degenerate. For example, one can easily see that this assumption holds with $\theta=1$ for any Lipschitz $u_{0}$ when $F(x, p, X)=|p|+1$.

Remark 4.7. As mentioned in Remark 4.4, additional continuity assumption on $u$ or $v$ in $\bar{\Omega}$ is usually needed to prove the comparison principle. However, we are not able to obtain uniform continuity of $\bar{u}$ and $\underline{u}$. Although we can show that $u^{\varepsilon}(\cdot, t)$ is continuous in $\bar{\Omega}$ for 
any fixed $t \geq 0$, it is not clear to us how to prove equi-continuity of $u^{\varepsilon}$ in $\bar{\Omega}$ with respect to $\varepsilon$, due to the regularity loss of $\eta_{\varepsilon}$ when $\varepsilon \rightarrow 0$.

Proof of Theorem 4.5. It is shown in Proposition 3.2 that $\bar{u}$ and $\underline{u}$ are respectively a subsolution and a supersolution of (1.1) in the interior of the domain. Let us focus on the verification of the state constraint boundary condition for $\underline{u}$.

Let $\theta$ be as in (4.6). We claim that

$$
u^{\varepsilon}(x, t) \leq u_{0}(x)-\frac{1}{2} \theta N \varepsilon^{2} \quad \text { for any }(x, t) \in \bar{\Omega}_{\varepsilon^{\mu}} \times(0, \infty) \text { and } \varepsilon>0 \text { small, }
$$

where $\mu>0$ appeared in (1.12) and $N=\left[t / \varepsilon^{2}\right]$.

To prove this claim, let us use the standard mollification to regularize $\eta_{\varepsilon}$ and $u_{0}$. By definition of $\eta_{\varepsilon}$ in (1.12), we can take a smooth function in $\bar{\Omega}$, still denoted by $\eta_{\varepsilon}$, such that $0 \leq \eta_{\varepsilon} \leq 1$ in $\bar{\Omega}, \eta_{\varepsilon}=1$ in $\bar{\Omega}_{\varepsilon^{\mu}}$ and $\eta_{\varepsilon}=0$ on $\partial \Omega$. We may also find a sequence $\psi_{j} \in \Psi_{\tau_{j}}$ such that $\tau_{j} \rightarrow 0$ and $\psi_{j} \rightarrow u_{0}$ uniformly in $\bar{\Omega}$ as $j \rightarrow \infty$.

Following the same argument as in the proof of Theorem 4.3, we use Lemma 3.1 and (4.6) to obtain that

$$
u^{\varepsilon}\left(x, \varepsilon^{2}\right) \leq \psi_{j}(x)-\theta \varepsilon^{2} F\left(x, \nabla \psi_{j}(x), \nabla^{2} \psi_{j}(x)\right)+o\left(\varepsilon^{2}\right) \leq \psi_{j}(x)-\theta \varepsilon^{2} \eta_{\varepsilon}^{2}+o\left(\varepsilon^{2}\right)
$$

for any $x \in \bar{\Omega}$. Since $\psi_{j}(x)-\theta \varepsilon^{2} \eta_{\varepsilon}^{2}$ is a smooth function below $u_{0}$, we may apply Lemma 3.1 and (4.6) again to obtain that, at any $x \in \bar{\Omega}$,

$$
u^{\varepsilon}\left(x, 2 \varepsilon^{2}\right) \leq \psi_{j}(x)-2 \theta \varepsilon^{2} \eta_{\varepsilon}^{2}+o\left(\varepsilon^{2}\right)
$$

Iterating this argument, we are led to

$$
u^{\varepsilon}(x, t) \leq \psi_{j}(x)-\theta \varepsilon^{2} N \eta_{\varepsilon}^{2},
$$

for all $(x, t) \in \bar{\Omega}_{\varepsilon^{\mu}} \times[0, \infty)$, which implies that (4.7) holds by letting $j \rightarrow \infty$.

We next show that $\underline{u}$ is a state constraint supersolution of (1.1). It suffices to verify that it is a supersolution on the boundary. Suppose that there is a test function $\phi \in$ $C^{2}(\bar{\Omega} \times(0, \infty))$ such that $\underline{u}-\phi$ attains a strict minimum at some $\left(x_{0}, t_{0}\right) \in \partial \Omega \times(0, \infty)$. Then as in the proof of Proposition 3.2, we can use the definition of $\underline{u}$ to find $r>0$ and $\left(x_{\varepsilon}, t_{\varepsilon}\right) \in \bar{\Omega} \times(0, \infty)$ such that $\left(x_{\varepsilon}, t_{\varepsilon}\right) \rightarrow\left(x_{0}, t_{0}\right)$ as $\varepsilon \rightarrow 0$ and

$$
\left(u^{\varepsilon}-\phi\right)\left(x_{\varepsilon}, t_{\varepsilon}\right) \leq \inf _{(x, t) \in B_{r}\left(x_{0}, t_{0}\right)}\left(u^{\varepsilon}-\phi\right)(x, t)+\varepsilon^{3} .
$$

It then follows from (4.7) that

$$
\left(u^{\varepsilon}-\phi\right)\left(x_{\varepsilon}, t_{\varepsilon}\right) \leq \inf _{\substack{(x, t) \in B_{r}\left(x_{0}, t_{0}\right) \\ x \in \bar{\Omega}_{\varepsilon} \mu}} u_{0}(x)-\phi(x, t)-\frac{1}{4} \varepsilon^{2} N
$$

when $\varepsilon>0$ is sufficiently small.

We next claim that $x_{\varepsilon} \notin \partial \Omega$ for all $\varepsilon>0$ small. If $x_{\varepsilon} \in \partial \Omega$, then we can find $x_{\varepsilon}^{\prime} \in \bar{\Omega}_{\varepsilon^{\mu}}$ and $\left(x_{\varepsilon}^{\prime}, t_{\varepsilon}\right) \in B_{r}\left(x_{0}, t_{0}\right)$ such that $\left|x_{\varepsilon}-x_{\varepsilon}^{\prime}\right|=\varepsilon^{\mu}$. Hence, we have

$$
\begin{aligned}
\inf _{\substack{(x, t) \in B_{r}\left(x_{0}, t_{0}\right) \\
x \in \bar{\Omega}_{\varepsilon} \mu}} & u_{0}(x)-\phi(x, t)-\frac{1}{4} \varepsilon^{2} N \\
& \leq u_{0}\left(x_{\varepsilon}^{\prime}\right)-\phi\left(x_{\varepsilon}^{\prime}, t_{\varepsilon}\right)-\frac{1}{4} \varepsilon^{2} N \\
& \leq u_{0}\left(x_{\varepsilon}\right)-\phi\left(x_{\varepsilon}, t_{\varepsilon}\right)-\frac{1}{4} \varepsilon^{2} N+L \varepsilon^{\mu},
\end{aligned}
$$


where $L>0$ is the sum of Lipschitz constants of $u_{0}$ and $\phi\left(\cdot, t_{\varepsilon}\right)$ in $\bar{\Omega}$.

On the other hand, since $u^{\varepsilon}=u_{0}$ on $\partial \Omega \times[0, \infty)$, we have

$$
\left(u^{\varepsilon}-\phi\right)\left(x_{\varepsilon}, t_{\varepsilon}\right) \geq u_{0}\left(x_{\varepsilon}\right)-\phi\left(x_{\varepsilon}, t_{\varepsilon}\right),
$$

which, together with (4.9), contradicts (4.8).

Now that $x_{\varepsilon} \in \Omega$ for all $\varepsilon>0$ small, the rest of the proof is the same as the argument in the interior case and finally we get

$$
\partial_{t} \phi\left(x_{0}, t_{0}\right)+F\left(x_{0}, \nabla \phi\left(x_{0}, t_{0}\right), \nabla^{2} \phi\left(x_{0}, t_{0}\right)\right) \geq 0 .
$$

In view of Proposition 3.3, the convergence of $u^{\varepsilon}$ in $\Omega \times[0, \infty)$ is an immediate consequence if the comparison principle (CP2) is available.

Let us give a simple example in the first order case.

Example 4.8. Our example is in one space dimension. Consider

$$
\partial_{t} u+\left|u_{x}\right|-|x|+2=0 \quad \text { in }(-1,1) \times(0, \infty)
$$

with

$$
u(x, 0)=u_{0}(x)=\frac{1}{2} x^{2} \quad \text { for } x \in[-1,1] .
$$

It is not difficult to verify that

$$
u(x, t)=\frac{1}{2} x^{2}-2 t
$$

is a solution with $\partial_{t} u=0$ satisfied in the viscosity sense. Moreover, due to [10, Theorem V.1] it is actually the unique state constraint solution that is continuous in $[-1,1] \times[0, \infty)$.

By direct calculations using Lemma 3.1, we have the game value in this case

$$
u^{\varepsilon}(x, t)=\frac{1}{2} x^{2}-2 N \varepsilon^{2}+o(1) \quad \text { for }(x, t) \in\left(-1+\varepsilon^{\mu}, 1-\varepsilon^{\mu}\right) \times[0, \infty)
$$

which implies that $u^{\varepsilon} \rightarrow u$ locally uniformly in $(-1,1) \times(0, \infty)$ as $\varepsilon \rightarrow 0$.

Note that $u^{\varepsilon}( \pm 1, t)=1 / 2$ for any $t \geq 0$, which implies that $\bar{u}( \pm 1, t) \geq 1 / 2$ for all $t \geq 0$. Sice $\underline{u}(x, t)=x^{2} / 2-2 t$ for $(x, t) \in[-1,1] \times(0, \infty)$, it is easily seen that $\bar{u}$ and $\underline{u}$ do not agree on $\partial \Omega \times(0, \infty)$. This suggests that, for any $T>0$, one cannot expect $u^{\varepsilon} \rightarrow u$ uniformly in $\bar{\Omega} \times[0, T]$.

4.2. Connection with the Neumann-type problem. Game interpretations of Neumanntype boundary problems for various nonlinear elliptic and parabolic equations are studied in $[11,30,3,14]$ etc.

We can slightly modify the game for (DBP1) described in Section 1.3 to provide an alternative game-theoretic approach to the following oblique boundary problem

$$
(\mathrm{OP}) \begin{cases}\partial_{t} u+F\left(x, \nabla u, \nabla^{2} u\right)=0 & \text { in } \Omega \times(0, \infty), \\ \left\langle\nabla u, \gamma_{0}\right\rangle=0 & \text { on } \partial \Omega \times(0, \infty), \\ u(\cdot, 0)=u_{0} & \text { in } \bar{\Omega},\end{cases}
$$

where $\gamma_{0}$ is a given oblique outward unit normal vector to $\partial \Omega$. We assume that $\gamma_{0}$ satisfies (H1) and (H2).

Indeed, in the game setting we set $A$ to be a singleton $\{0\}$, let $f \equiv 0$ and take $g(x, 0)=$ $g(x)$ for all $x \in \partial \Omega$. We assume that $\gamma_{0}$ satisfies (H1) and (H2). Moreover, we also need 
to change the game rules: fix $\tau \in(0,1)$ and replace $\zeta_{\varepsilon}$ in (1.14) and in (1.15) by $\varepsilon^{-\tau} \zeta_{\varepsilon}$, i.e., we set

$$
\begin{gathered}
h_{k}=\sqrt{2} \varepsilon \eta_{\varepsilon}\left(x_{k}\right) v_{k}-\varepsilon^{2-\tau} \zeta_{\varepsilon}\left(x_{k}\right) \gamma_{0}\left(x_{k}\right) \\
l_{k}=\sqrt{2} \varepsilon \eta_{\varepsilon}\left(x_{k}\right)\left\langle p_{k}, v_{k}\right\rangle+\varepsilon^{2} \eta_{\varepsilon}^{2}\left(x_{k}\right)\left(\left\langle X_{k} v_{k}, v_{k}\right\rangle+F\left(y_{k}, p_{k}, X_{k}\right)\right)+\varepsilon^{2-\tau} \zeta_{\varepsilon} g\left(x_{k}\right) .
\end{gathered}
$$

One should view such changes as an emphasis on the boundary game activity; the order was reduced from $\varepsilon^{2}$ to $\varepsilon^{2-\tau}$.

Despite these changes, the whole argument of the derivation in the previous section will work. We therefore obtain the following variant of (1.29):

$$
\begin{array}{rl}
\partial_{t} u^{\varepsilon}\left(x_{\varepsilon}, t_{\varepsilon}\right)+\eta_{\varepsilon}^{2} & F\left(x_{\varepsilon}, \nabla u^{\varepsilon}\left(x_{\varepsilon}, t_{\varepsilon}\right), \nabla^{2} u^{\varepsilon}\left(x_{\varepsilon}, t_{\varepsilon}\right)\right) \\
& +\varepsilon^{-\tau} \zeta_{\varepsilon}\left(\left\langle\nabla u^{\varepsilon}\left(x_{\varepsilon}, t_{\varepsilon}\right), \gamma_{0}\left(x_{\varepsilon}\right)\right\rangle-g\left(x_{\varepsilon}\right)\right)=o(1) .
\end{array}
$$

(1) If $\varepsilon^{-\tau} \zeta_{\varepsilon} \rightarrow \infty$ as $\varepsilon \rightarrow 0$, we end up with the oblique boundary condition (4.10).

(2) If $\varepsilon^{-\tau} \zeta_{\varepsilon} \rightarrow 0$ as $\varepsilon \rightarrow 0$, then we get the equation (1.1) on $\partial \Omega \times(0, \infty)$.

(3) A mixed case shows up when $\varepsilon^{-\tau} \zeta_{\varepsilon} \rightarrow c$ for $c>0$.

We therefore can obtain the following result for (NP).

Theorem 4.9 (Neumann-type boundary problem). Assume that $\Omega \subset \mathbb{R}^{n}$ is a bounded $C^{2}$ domain. Assume that (F1) and (F2) hold. Let $\gamma_{0}$ be an oblique outward unit normal vector to $\partial \Omega$ satisfying (H1) and (H2) with $A=\{0\}$. Let $u_{0}$ and $g$ be Lipschitz in $\bar{\Omega}$. Assume (1.20)-(1.24). For any $\varepsilon>0$ small, let $u^{\varepsilon}$ be the value function defined as in (1.16) for the game with $A=\{0\}, f \equiv 0$ in $\bar{\Omega}$ and $\zeta_{\varepsilon}$ replaced by $\varepsilon^{-\tau} \zeta_{\varepsilon}$ with $0<\tau<1$. Then $\bar{u}$ and $\underline{u}$ given in (1.25) are respectively a subsolution and a supersolution of $(O P)$. Moreover, if the comparison principle for $(O P)$ holds, then $u^{\varepsilon} \rightarrow u$ locally uniformly in $\bar{\Omega} \times[0, \infty)$ as $\varepsilon \rightarrow 0$, where $u$ is a unique solution of $(O P)$.

It is clear that when $\gamma_{0}=\nu$, this result reduces to a game-theoretic interpretation for the Neumann problem (NP).

It seems that one cannot take $\tau=1$ for the game interpretation above; when $\tau=1$, in (4.13) there is an extra term $\left\langle\nabla^{2} u^{\varepsilon} \gamma_{0}, \gamma_{0}\right\rangle$ coming into play. We are not able to remove this term in our expansion.

Remark 4.10. It is worth pointing out that our game construction for Neumann boundary conditions here is still different from those in [14], though the target equation is the same. The main difference is that in (4.11) we maintain a "push" at the direction of $-\gamma_{0}$ with magnitude of order $\varepsilon^{2-\tau}$ while in the game described in [14] such a term (but of order $\varepsilon$ ) appears as a projection to $\partial \Omega$, depending on how far the game position is away from $\bar{\Omega}$ if an exit occurs in the previous step. Our interpretation of Neumann boundary conditions is also different from that in $[36,30]$, where reflections on $\partial \Omega$ are employed.

\section{Fast eVolution asymptotics And ELLIPTiC PROBlems}

In this section, we intend to adopt the game-theoretic approach to study the asymptotic behavior of the solution to (DBP2) as the given parameter $\delta$ tends to 0 . We assume that $H$ still has the form (1.8) and all assumptions in Theorem 1.1 still hold.

Using the game described in Section 1.3, we can construct a family of games whose value functions $u^{\varepsilon, \delta}$ converge, as $\varepsilon \rightarrow 0$, to the unique solution of (DBP2), denoted by $u_{\delta}$. In fact, in this case one can simply replace the running cost $F$ in (1.15) by $F / \delta$ in the 
game introduced in Section 1.3 and then still take $u^{\varepsilon, \delta}$ to be the value function defined by (1.16).

The dynamic programming principle for the new game is as below:

$$
u^{\varepsilon, \delta}(x, t)=\min _{p, X} \max _{a, v}\left\{u^{\varepsilon, \delta}\left(x+h, t-\varepsilon^{2}\right)-l_{\delta}\right\},
$$

where $|p| \leq \varepsilon^{-\alpha},|X| \leq \varepsilon^{-\beta}, a \in A$ and $|v| \leq \varepsilon^{-\gamma}$, and $h$ is given by (1.18) and

$$
l_{\delta}=\sqrt{2} \varepsilon \eta_{\varepsilon}(x)\langle p, v\rangle+\varepsilon^{2} \eta_{\varepsilon}^{2}(x)\left(\langle X v, v\rangle+\frac{1}{\delta} F(x, p, X)\right)+\varepsilon^{2} \zeta_{\varepsilon}(x) g(x, a) .
$$

It is clear that we need to assume $\delta \geq \varepsilon^{\sigma}$ with $\sigma$ satisfying (1.38) to guarantee the smallness of $l_{\delta}$ as $\varepsilon \rightarrow 0$.

We are interested in the behavior of $u^{\varepsilon, \delta}$ as $(\varepsilon, \delta) \rightarrow 0$, since this will also give us asymptotic behavior for $u_{\delta}$ as $\delta \rightarrow 0$. This part of our work is inspired by recent results on similar asymptotic problems for the eikonal equation [1] and heat equations [25] with linear dynamical boundary conditions. In the context of diffusion problems, limiting behavior of $u_{\delta}$ as $\delta \rightarrow 0$ for parabolic equations is called large diffusion behavior [25]. As shown in $[1,25]$, one can expect that $u_{\delta}$ converges to $U$ as $\delta \rightarrow 0$, where $U$ solves (DBP3).

Here $u_{\infty}$ is obtained as the large-time limit of the solution of (DP1) with the Dirichlet condition (1.30). The problem (DBP3) can be viewed as an (interior) stationary version of (DBP1). The appearance of $u_{\infty}$ reveals the existence of an initial layer in the limit process for (DBP2) as $\delta \rightarrow 0$. A simple example to show such asymptotics will be given in Section 5.1 below.

We actually give a slightly stronger result by investigating the limit of our game value $u^{\varepsilon, \delta}$ for (DBP2) as $\varepsilon \rightarrow 0$ and $\delta \rightarrow 0$ simultaneously. It turns out that, under proper assumptions on $F$ and requirements on the vanishing rate on $\delta(\varepsilon)$, we can obtain a limit $U$ of $u^{\varepsilon, \delta}$, which uniquely solves (DBP3). Moreover, by considering such a joint limit for $\varepsilon$ and $\delta$, we can obtain a game interpretation for fully nonlinear elliptic equations with dynamic boundary conditions.

5.1. An example of large diffusion behavior. Let us start with a typical example on the behavior of the solution of (DBP2) as $\delta \rightarrow 0$.

Example 5.1. Let us consider the following linear problem in one space dimension:

$$
\text { (E) } \begin{cases}\delta \partial_{t} u-u_{x x}=1 & \text { in }(-1,1) \times(0, \infty), \\ \partial_{t} u+u_{x}=0 & \text { on }\{1\} \times(0, \infty), \\ \partial_{t} u-u_{x}=0 & \text { on }\{-1\} \times(0, \infty), \\ u(\cdot, 0)=u_{0} & \text { in }[-1,1],\end{cases}
$$

where $u_{0}$ is a given Lipschitz function in $[-1,1]$ satisfying $u_{0}( \pm 1)=0$. We can show that the unique solution $u_{\delta} \rightarrow U$ locally uniformly in $[-1,1] \times(0, \infty)$ as $\delta \rightarrow 0$, where

$$
U(x, t)=\frac{1}{2}\left(1-x^{2}\right)+t
$$

for all $(x, t) \in[-1,1] \times[0, \infty)$.

Indeed, since $u_{0}$ is Lipschitz in $[-1,1]$ and satisfies $u_{0}( \pm 1)=0$, we can find $c_{0}>1$ such that for any $x \in[-1,1]$

$$
\left|u_{0}(x)\right| \leq \frac{c_{0}}{2}\left(1-x^{2}\right) .
$$


Letting

$$
t_{1}=\frac{\delta}{2} \ln \frac{c_{0}-1}{\delta}, \quad t_{2}=\frac{\delta}{2} \ln \frac{1+c_{0}}{\delta}
$$

for any $\delta \in\left(0, c_{0}-1\right)$, one can show that

$$
w_{\delta,+}(x, t)= \begin{cases}\frac{1}{2}\left(\left(1-c_{0}\right) e^{-\frac{2 t}{\delta}}-1\right)\left(x^{2}-1\right)+2 c_{0} t & \text { if } 0 \leq t \leq t_{1} \\ \frac{1}{2}(\delta+1)\left(1-x^{2}\right)+(\delta+1) t+\left(2 c_{0}-\delta-1\right) t_{1} & \text { if } t>t_{1}\end{cases}
$$

and

$$
w_{\delta,-}(x, t)= \begin{cases}\frac{1}{2}\left(\left(1+c_{0}\right) e^{-\frac{2 t}{\delta}}-1\right)\left(x^{2}-1\right)-2 c_{0} t & \text { if } 0 \leq t \leq t_{2} \\ \frac{1}{2}(\delta-1)\left(x^{2}-1\right)+t-\left(2 c_{0}+1\right) t_{2} & \text { if } t>t_{2}\end{cases}
$$

are respectively a continuous supersolution and a continuous subsolution of (E). By comparison principle for (DBP1), we get

$$
w_{\delta,-} \leq u_{\delta} \leq w_{\delta,+} \quad \text { in }[-1,1] \times[0, \infty) .
$$

Since $w_{\delta, \pm} \rightarrow U$ locally uniformly in $[-1,1] \times(0, \infty)$ as $\delta \rightarrow 0$, we obtain the convergence $u_{\delta} \rightarrow U$ as well.

Let us denote

$$
u_{\infty}(x)=\frac{1}{2}\left(1-x^{2}\right)
$$

We can easily verify that $U$ solves the following dynamic boundary problem

$$
\begin{cases}-U_{x x}=1 & \text { in }(-1,1) \times(0, \infty), \\ \partial_{t} U+U_{x}=0 & \text { on }\{1\} \times(0, \infty), \\ \partial_{t} U-U_{x}=0 & \text { on }\{-1\} \times(0, \infty), \\ U(\cdot, 0)=u_{\infty} & \text { in }[-1,1],\end{cases}
$$

and $u_{\infty}$ is the large time limit of the solution to

$$
\begin{cases}\partial_{t} u-u_{x x}=1 & \text { in }(-1,1) \times(0, \infty), \\ u=0 & \text { on }\{ \pm 1\} \times(0, \infty) \\ u(\cdot, 0)=u_{0} & \text { in }[-1,1]\end{cases}
$$

5.2. Comparison principles. As is mentioned before, we aim to show that the game value $u^{\varepsilon, \delta}$ for (DBP1) converges to a unique solution $U$ of (DBP3) as $\varepsilon, \delta \rightarrow 0$. To this end, we need to prepare two comparison principles. One is for the Dirichlet problem (DP2), which is used to uniquely determine the initial value $u_{\infty}$ of (DBP3). The other one is for (DBP3), which is already stated in Theorem 2.5. It gives the uniqueness of solutions to (DBP3) and enables us to conclude the convergence of $u^{\varepsilon, \delta}$.

A comparison result for (DP2) allows us to find the large-time profile $u_{\infty}$ for (DP1). One difficultly lies at a possible loss of the Dirichlet boundary data in (DP2). In order to overcome this difficulty, we again assume (F5) for the existence of boundary barriers so that (1.30) can be interpreted in the strict sense.

We remark that (F5) is in general quite restrictive and $u_{\infty}$ may be uniquely determined as the large-time profile for (DP1) under weaker assumptions. In [1], although the equation and boundary conditions are more specific, no assumptions like (F5) are imposed there and the large-time behavior for (DP1) is investigated with (1.30) interpreted in the viscosity sense. 
Theorem 5.2 (Comparison principle for stationary Dirichlet problems). Assume that $\Omega \subset \mathbb{R}^{n}$ is a bounded domain. Assume that (F3) and (F4) hold. Let $u \in U S C(\bar{\Omega})$ and $v \in L S C(\bar{\Omega})$ be respectively a bounded subsolution and a bounded supersolution of (1.36). If $u \leq v$ on $\partial \Omega$ then $u \leq v$ in $\bar{\Omega}$.

We refer to [32] for such a result in the first order case. It is not difficult to see that the operator in Example 5.1 satisfies the assumptions (F3) and (F4). The idea of proving this comparison result under the assumption (F4) is to first compare the subsolution $u$ with a strict supersolution

$$
v_{j}:=\lambda_{j} v+\left|1-\lambda_{j}\right| \sup _{x \in \bar{\Omega}}|v(x)|
$$

of (DP2) and then let $\lambda_{j} \rightarrow 1$. As the proof is standard, we omit the details here.

Remark 5.3. The assumption (F4) is typically used to find a strict sub- or supersolution. There are certainly many other ways to do this. For example, (F4) actually fails to hold when $F$ is the Laplacian operator, i.e., $F(x, p, X)=-\operatorname{tr} X$. However, one can subtract a positive quadratic function from a supersolution to construct a strict supersolution; namely, if $v$ is a supersolution, then

$$
v_{\lambda}(x)=v(x)-\lambda|x|^{2}
$$

is a supersolution of $-\Delta v_{\lambda}=2 n \lambda$ in $\Omega \subset \mathbb{R}^{n}$ for any $\lambda>0$. Raising slightly the boundary data, we can get a strict supersolution of Dirichlet problem and proceed to the same comparison argument before sending $\lambda \rightarrow 0$.

We choose to impose (F4) for our convenience in providing a general result including the first order equations.

The following is an immediate consequence of Theorem 5.2.

Proposition 5.4 (Large-time asymptotics). Assume that $\Omega \subset \mathbb{R}^{n}$ is a bounded domain. Assume that (F3), (F4) and (F5) hold. Let $u_{0}$ be Lipschitz in $\bar{\Omega}$. Let $\tilde{u}$ be the unique viscosity solution of (DP1). Then

$$
\tilde{u}(\cdot, t) \rightarrow u_{\infty} \quad \text { uniformly in } \bar{\Omega} \text { as } t \rightarrow \infty,
$$

where $u_{\infty}$ is the unique solution of (DP2).

To show this result, one can rescale $\tilde{u}$ with a parameter $\lambda>0$ to get

$$
\tilde{v}_{\lambda}(x, t)=\tilde{u}(x, t / \lambda), \quad(x, t) \in \bar{\Omega} \times[0, \infty),
$$

which can be easily verified to be the solution of

$$
\lambda \partial_{t} \tilde{v}_{\lambda}+F\left(x, \nabla \tilde{v}_{\lambda}, \nabla^{2} \tilde{v}_{\lambda}\right)=0 \quad \text { in } \Omega \times(0, \infty)
$$

with $\tilde{v}_{\lambda}=u_{0}$ on $\bar{\Omega} \times\{0\}$ and on $\partial \Omega \times(0, \infty)$ under the assumption (F5). By the standard stability theory and the comparison principle for (DP2), it follows that $\tilde{v}_{\lambda}$ converges to $u_{\infty}$ locally uniformly in $\bar{\Omega} \times(0, \infty)$ as $\lambda \rightarrow 0$. This completes the proof of $(5.4)$.

On the other hand, Theorem 2.5 can be obtained by adapting the uniqueness arguments in [8]. Let us first give a comparison result for a subsolution and a strict supersolution. More precisely, for any given $c>0$, assuming that $u$ is a subsolution of (1.34) and (1.2) while $v$ is a supersolution of

$$
F\left(x, \nabla u, \nabla^{2} u\right)=c \quad \text { in } \Omega \times(0, \infty)
$$

with (1.2), we prove that $u \leq v$ in $\bar{\Omega} \times[0, \infty)$. 
Theorem 5.5 (Comparison principle for strict sub/supersolutions). Assume that $\Omega$ is a bounded $C^{2,1}$ domain in $\mathbb{R}^{n}$. Assume that (F3), (H1), (H2), (B1) and (B2) hold. Let $u \in U S C(\bar{\Omega} \times[0, \infty))$ be a subsolution of $(1.34)$ and $(1.2)$. Let $v \in L S C(\bar{\Omega} \times[0, \infty))$ be a supersolution of (5.5) and (1.2) with $c>0$. If $u \leq v$ on $\bar{\Omega} \times\{0\}$, then $u \leq v$ in $\bar{\Omega} \times[0, \infty)$.

Since comparison results like Theorem 5.5 are not explicitly available in the literature, we give a proof of Theorem 5.5 in Appendix A for the reader's convenience.

We can prove Theorem 2.5 with ease by utilizing the assumption (F4) and the comparison result in Theorem 5.5.

Proof of Theorem 2.5. For the given supersolution $v$, we take

$$
v_{j}(x, t)=\lambda_{j} v(x, t)+\left|1-\lambda_{j}\right| \sup _{x \in \bar{\Omega}}|v(x, 0)|, \quad \text { for all }(x, t) \in \bar{\Omega} \times[0, \infty) .
$$

In view of $(\mathrm{F} 4)$, it is not difficult to see that $v_{j}$ is a supersolution of (5.5) with $c=c_{j}>0$ and the dynamic boundary condition (1.2). Moreover, since

$$
v_{j}(x, 0) \geq v(x, 0), \quad \text { for any } x \in \bar{\Omega},
$$

we may apply Theorem 5.5 to deduce that

$$
u \leq v_{j} \quad \text { in } \bar{\Omega} \times[0, \infty) .
$$

Sending $j \rightarrow \infty$, we end up with $u \leq v$ in $\bar{\Omega} \times[0, \infty)$, as desired.

We conclude this section by emphasizing that Remark 5.3 also applies to elliptic equations with dynamic boundary conditions. The assumption (F4) is only a sufficient condition to find strict sub- and supersolutions of (DBP3). One can find a different way to construct sub- or supersolutions when (F4) does not hold.

5.3. Game approximation. We finally study limit behavior of $u^{\varepsilon, \delta}$ as $(\varepsilon, \delta) \rightarrow 0$ with a requirement on the dependence $\delta(\varepsilon)$. We first examine a general case under the weaker assumptions (F1), (F2) and (F5). Later we additionally impose (F3) and (F4) so as to use the comparison results in Section 5.2 to conclude the convergence of $u^{\varepsilon, \delta}$.

5.3.1. General results. Let us first discuss the general case when only assumptions (F1) and (F2) are imposed. In this case, besides the conditions (1.20)-(1.24) on $\alpha, \beta$ and $\gamma$, we need to assume that $\delta \geq \varepsilon^{\sigma}$ with $\sigma>0$ satisfying (1.38)-(1.41).

Taking these extra assumptions on $\sigma$ into consideration, we define a modified version of the half relaxed limits of $u^{\varepsilon, \delta}$ as $(\varepsilon, \delta) \rightarrow 0$ : for any $(x, t) \in \bar{\Omega} \times[0, \infty)$, let

$$
\begin{array}{r}
\bar{u}^{\sigma}(x, t)=\lim _{r \rightarrow 0} \sup \left\{u^{\varepsilon, \delta}(y, s):(y, s) \in \bar{\Omega} \times[0, \infty),|x-y|+|t-s|+\varepsilon+\delta \leq r,\right. \\
\delta \geq \varepsilon^{\sigma} \text { with } \sigma>0 \text { satisfying (1.38)-(1.41)\}, } \\
\underline{u}_{\sigma}(x, t)=\lim _{r \rightarrow 0} \inf \left\{u^{\varepsilon, \delta}(y, s):(y, s) \in \bar{\Omega} \times[0, \infty),|x-y|+|t-s|+\varepsilon+\delta \leq r,\right. \\
\left.\delta \geq \varepsilon^{\sigma} \text { with } \sigma>0 \text { satisfying (1.38)-(1.41) }\right\} .
\end{array}
$$

The boundedness of $u^{\varepsilon, \delta}$ uniformly in $(\varepsilon, \delta)$ will be justified later in Lemma 5.8. 
Theorem 5.6 (Half limits of game values). Assume that $\Omega \subset \mathbb{R}^{n}$ is a bounded $C^{2,1}$ domain. Assume that (F1), (F2), (F5), (H1), (H2), (B1) and (B2) hold. Assume that (1.20)-(1.24) hold. Assume that $u_{0}$ is Lipschitz in $\bar{\Omega}$. Let $\varepsilon, \delta>0$ and $u^{\varepsilon, \delta}$ be the game value in (1.16) but with running cost $F$ in (1.15) replaced by $F / \delta$. Let $\bar{u}^{\sigma}$ and $\underline{u}_{\sigma}$ be defined by (5.6). Then $\bar{u}^{\sigma}$ are $\underline{u}_{\sigma}$ are respectively a subsolution and a supersolution of (1.34) with dynamic boundary condition (1.2).

Remark 5.7. Concerning the condition $\delta \geq \varepsilon^{\sigma}$ in (5.6), it is equivalent to assume that there exists $C>0$ such that $\delta \geq C \varepsilon^{\sigma}$ for any $\varepsilon>0$ sufficiently small, or

$$
\liminf _{\varepsilon \rightarrow 0} \frac{\delta}{\varepsilon^{\sigma}}>0
$$

These weaker assumptions will not change our result in Theorem 5.10. We choose to keep our current form for simplicity of exhibition.

Let us prove uniform boundedness of $u^{\varepsilon, \delta}$ in $\varepsilon$ and $\delta$.

Lemma 5.8 (Uniform boundedness). Assume that $\Omega \subset \mathbb{R}^{n}$ is a bounded $C^{2,1}$ domain. Assume that (F1), (F2), (F5), (H1), (H2), (B1) and (B2) hold. Assume that $u_{0}$ is Lipschitz in $\bar{\Omega}$. Assume that (1.20)-(1.24) hold. Let $u^{\varepsilon, \delta}$ be the game value in (1.16) but with running cost $F$ in (1.15) replaced by $F / \delta$. Then for any $T>0, u^{\varepsilon, \delta}$ is bounded in $\bar{\Omega} \times[0, T]$ uniformly in $\varepsilon, \delta>0$.

Proof. Our proof relies on the Taylor expansion of (5.1) for smooth functions, as given by Lemma 3.1. Indeed, let us take an analogue of (3.1); more precisely, we define $S_{\delta}^{\varepsilon}$ : $C(\bar{\Omega}) \rightarrow C(\bar{\Omega})$ by

$$
S_{\delta}^{\varepsilon}[w](x)=\min _{p, X} \max _{a, v}\left\{w(x+h)-l_{\delta}\right\}
$$

for any $w \in C(\bar{\Omega})$, where $h$ and $l_{\delta}$ are respectively given by (1.18) and (5.2). It is clear that $S_{\delta}^{\varepsilon}$ is monotone.

Let us take a smooth function $\psi_{\tau}^{+}$as in (F5), for any $\tau>0$ and $x_{0} \in \partial \Omega$ arbitrarily taken, such that

$$
u_{0} \leq \psi_{\tau}^{+} \quad \text { in } \bar{\Omega}
$$

and

$$
F\left(x, \nabla \psi_{\tau}^{+}(x), \nabla^{2} \psi_{\tau}^{+}(x)\right) \geq 0 \text { for any } x \in \Omega .
$$

Applying Lemma 3.1 with $\varphi=\psi_{\tau}^{+}$for $S_{\delta}^{\varepsilon}$ instead of $S^{\varepsilon}$, we have

$$
\begin{aligned}
u^{\varepsilon, \delta}\left(x, \varepsilon^{2}\right)= & S_{\delta}^{\varepsilon}\left[u_{0}\right](x) \leq S_{\delta}^{\varepsilon}\left[\psi_{\tau}^{+}\right](x) \\
& \leq \psi_{\tau}^{+}(x)-\frac{\varepsilon^{2} \eta_{\varepsilon}^{2}(x)}{\delta} F\left(x, \nabla \psi_{\tau}^{+}(x), \nabla^{2} \psi_{\tau}^{+}(x)\right)-\varepsilon^{2} \zeta_{\varepsilon}(x) H\left(x, \nabla \psi_{\tau}^{+}(x)\right)+o\left(\varepsilon^{2}\right) \\
& \leq \psi_{\tau}^{+}(x)-\varepsilon^{2} \zeta_{\varepsilon}(x) H\left(x, \nabla \psi_{\tau}^{+}(x)\right)+o\left(\varepsilon^{2}\right) .
\end{aligned}
$$

Since $\zeta_{\varepsilon}(x) H\left(x, \nabla \psi_{\tau}^{+}(x)\right)$ is uniformly bounded for all $x \in \bar{\Omega}$ and $\varepsilon>0$ small, we have

$$
u^{\varepsilon}\left(x, \varepsilon^{2}\right) \leq \psi_{\tau}^{+}(x)+\varepsilon^{2} M_{\tau}+o\left(\varepsilon^{2}\right),
$$

where $M_{\tau}>0$ and the modulus in the error depend only on $H$ and $\psi_{\tau}^{+}$. By iteration, it follows that

$$
u^{\varepsilon, \delta}(\cdot, t) \leq \psi_{\tau}^{+}+M_{\tau} t+\omega(1) \quad \text { in } \bar{\Omega} \text { for all } t \geq 0
$$

for a modulus of continuity $\omega$ independent of $\varepsilon, \delta$, which shows the boundedness of $u^{\varepsilon, \delta}$ from above uniformly in $\varepsilon$ and $\delta$. A lower bound can be obtained in a symmetric manner. 
Lemma 5.9 (Consistency for elliptic problems). Suppose that $\Omega \subset \mathbb{R}^{n}$ is a bounded $C^{2}$ domain. Assume that (H1), (H2), (F1), (F2), (B1) and (B2) hold. Let $H$ be given by (1.8). Assume that (1.20)-(1.24) hold and $\delta \geq \varepsilon^{\sigma}$ with $\sigma>0$ satisying (1.38)-(1.41). Let $S_{\delta}^{\varepsilon}$ be given by (5.7). Then for any $\varphi \in C^{2}(\bar{\Omega})$ and $x \in \bar{\Omega}$,

$$
\begin{array}{r}
S_{\delta}^{\varepsilon}[\varphi](x)-\varphi(x)=-\varepsilon^{2} \delta^{-1} \eta_{\varepsilon}^{2}(x) F\left(x, \nabla \varphi(x), \nabla^{2} \varphi(x)\right)-\varepsilon^{2} \zeta_{\varepsilon}(x) H(x, \nabla \varphi(x)) \\
+o\left(\varepsilon^{2} \delta^{-1} \eta_{\varepsilon}^{2}(x)+\varepsilon^{2}\right) .
\end{array}
$$

The proof is quite similar to that of Lemma 3.1. However, since the estimate (5.9) involves the additional parameter $\delta$ with more conditions, we below give full details, explaining how to use the condition $\delta \geq \varepsilon^{\sigma}$ and (1.38)-(1.41).

Proof of Lemma 5.9. For any $\varphi \in C^{2}(\bar{\Omega}), x \in \bar{\Omega}$ and $\delta \geq \varepsilon^{\sigma}$, by Taylor expansion we have

$$
S^{\varepsilon}[\varphi](x)-\varphi(x)=\min _{p, X} \max _{a, v}\left\{\langle\nabla \varphi(x), h\rangle+\frac{1}{2}\left\langle\nabla^{2} \varphi(x) h, h\right\rangle-l_{\delta}\right\}+o\left(\varepsilon^{2}\right),
$$

where $h$ and $l_{\delta}$ are given as in (1.18) and (5.2). As mentioned before, (1.38) is used to guarantee that $l_{\delta} \rightarrow 0$ as $\varepsilon \rightarrow 0$. The condition (1.20) is adopted to deduce that $|h|^{3}$ is of order $o\left(\varepsilon^{2}\right)$. We write the right hand side of (5.10) as $Q_{1}+Q_{2}$, where

$$
\begin{aligned}
Q_{1}:=\min _{p, X} \max _{v}\left\{\sqrt{2} \varepsilon \eta_{\varepsilon}(x)\langle\nabla \varphi(x)-p, v\rangle+\varepsilon^{2} \eta_{\varepsilon}^{2}(x)\right. & \left\langle\left(\nabla^{2} \varphi(x)-X\right) v, v\right\rangle \\
& \left.-\varepsilon^{2} \delta^{-1} \eta_{\varepsilon}^{2}(x) F(x, p, X)\right\}
\end{aligned}
$$

and $Q_{2}$ is the same as that in (3.5).

Similar to the proof of Lemma 3.1, our next step is to show that

$$
Q_{1}=-\varepsilon^{2} \delta^{-1} \eta_{\varepsilon}^{2}(x) F\left(x, \nabla \varphi(x), \nabla^{2} \varphi(x)\right)+o\left(\varepsilon^{2} \delta^{-1} \eta_{\varepsilon}^{2}\right) .
$$

The proof for " $\leq$ " is based on the choice $p=\nabla \varphi(x)$ and $X=\nabla^{2} \varphi(x, t)$ in (3.3). It suffices to show that " $\geq$ " holds.

The conditions (1.22) and (1.39) allow us to take $q$ such that

$$
0<q<\min \left\{1-\gamma-\beta, 1+\gamma-\sigma-\beta \sigma_{2}\right\} .
$$

We discuss the following cases.

a) Suppose $|\nabla \varphi(x)-p| \leq \varepsilon^{q}$ and $\nabla^{2} \varphi(x) \leq X+\varepsilon^{\gamma} I$. Then we may take $v=0$ so that

$$
\begin{aligned}
Q_{1} & \geq-\varepsilon^{2} \delta^{-1} \eta_{\varepsilon}^{2}(x) F(x, p, X) \\
& \geq-\varepsilon^{2} \delta^{-1} \eta_{\varepsilon}^{2}(x) F\left(x, \nabla \varphi(x), \nabla^{2} \varphi(x)\right)-L \varepsilon^{2} \delta^{-1} \eta_{\varepsilon}^{2}(x)\left(\varepsilon^{q}+\varepsilon^{\gamma}\right),
\end{aligned}
$$

where $L=L_{F}(R)$ is the local Lipschitz constant of $F$ in (F1) for $R=1+\|\varphi\|_{C^{2}(\bar{\Omega})}$. This yields (5.12) immediately.

b) Suppose $|\nabla \varphi(x)-p| \leq \varepsilon^{q}$ and $X+\varepsilon^{\gamma} I-\nabla^{2} \varphi(x)$ is not positive semi-definite. Denote by $\lambda$ the maximum positive eigenvalue of $\nabla^{2} \varphi(x)-X-\varepsilon^{\gamma} I$. Let $v$ be an eigenvector associated to the eigenvalue $\lambda$ with $|v|=\varepsilon^{-\gamma}$. Moreover, we may adjust the sign of $v$ such that

$$
\langle\nabla \varphi(x)-p, v\rangle \geq 0
$$


By the Lipschitz continuity (F1) of $F$ and the growth condition (F2) again, it follows that

$$
\begin{aligned}
Q_{1} \geq-\varepsilon^{2} \delta^{-1} \eta_{\varepsilon}^{2}(x) F\left(x, \nabla \varphi(x), \nabla^{2} \varphi(x)-\lambda I\right)+\varepsilon^{2} \eta_{\varepsilon}^{2}(x)\left(\lambda \varepsilon^{-2 \gamma}+\varepsilon^{-\gamma}\right) \\
-L \varepsilon^{2} \delta^{-1} \eta_{\varepsilon}^{2}(x)\left(\varepsilon^{q}+\varepsilon^{\gamma}\right),
\end{aligned}
$$

which yields

$$
Q_{1} \geq-\varepsilon^{2} \delta^{-1} \eta_{\varepsilon}^{2}(x) F\left(x, \nabla \varphi(x), \nabla^{2} \varphi(x)\right)+\varepsilon^{2} \eta_{\varepsilon}^{2}(x)\left(\lambda \varepsilon^{-2 \gamma}-C \delta^{-1} \lambda^{\sigma_{2}}\right)-o\left(\varepsilon^{2} \delta^{-1} \eta_{\varepsilon}^{2}(x)\right)
$$

for some $C>0$ large. Noticing that $\lambda \leq C\left(1+\varepsilon^{-\beta}\right)$ and $\delta \geq \varepsilon^{\sigma}$, we have

$$
\lambda \varepsilon^{-2 \gamma}-C \delta^{-1} \lambda^{\sigma_{2}} \geq \lambda\left(\varepsilon^{-2 \gamma}-C \varepsilon^{-\sigma-\beta\left(\sigma_{2}-1\right)}\right) \geq 0
$$

when $\varepsilon>0$ is small, since (1.41) holds. We therefore get (5.12) in this case too.

c) Suppose that $|\nabla \varphi(x)-p|>\varepsilon^{q}$. Take $v$ parallel to $\nabla \varphi(x)-p$ with $|v|=\varepsilon^{-\gamma}$. Then, by (F2), we get

$$
Q_{1} \geq \sqrt{2} \varepsilon^{1-\gamma} \eta_{\varepsilon}(x)|\nabla \varphi(x)-p|-C \eta_{\varepsilon}^{2}(x)\left(\varepsilon^{2-\beta-2 \gamma}+\delta^{-1} \varepsilon^{2-\beta \sigma_{2}}\right)-C \varepsilon^{2} \delta^{-1} \eta_{\varepsilon}^{2}(x)|p|^{\sigma_{1}},
$$

for some $C>0$ and $\varepsilon>0$ small, which, in view of (5.13), implies that

$$
Q_{1} \geq \varepsilon^{1-\gamma} \eta_{\varepsilon}(x)|\nabla \varphi(x)-p|-C \varepsilon^{2} \delta^{-1} \eta_{\varepsilon}^{2}(x)|p|^{\sigma_{1}},
$$

due to the assumption $\delta \geq \varepsilon^{\sigma}$.

If $|p| \leq 2|\nabla \varphi(x)|$, then for $\varepsilon>0$ small we have

$$
\begin{aligned}
\varepsilon^{1-\gamma} \eta_{\varepsilon}(x)|\nabla \varphi(x)-p|-C \varepsilon^{2} \delta^{-1} \eta_{\varepsilon}^{2}(x)|p|^{\sigma_{1}} & \geq \varepsilon^{1-\gamma} \eta_{\varepsilon}(x)|\nabla \varphi(x)-p|-O\left(\varepsilon^{2} \delta^{-1} \eta_{\varepsilon}^{2}(x)\right) \\
& \geq \frac{1}{2} \varepsilon^{1-\gamma+q} \eta_{\varepsilon}^{2}(x) .
\end{aligned}
$$

The last inequality comes from the fact that $1-\gamma+q<2-\sigma$ due to (5.13) again.

If $|p|>2|\nabla \varphi(x)|$, which yields $|p| \geq 2 \varepsilon^{q} / 3$, then

$$
\begin{aligned}
\varepsilon^{1-\gamma} \eta_{\varepsilon}|\nabla \varphi(x)-p|-C \varepsilon^{2} \delta^{-1} \eta_{\varepsilon}^{2}|p|^{\sigma_{1}} & \geq \frac{1}{2} \varepsilon^{1-\gamma} \eta_{\varepsilon}|p|-C \varepsilon^{2} \delta^{-1} \eta_{\varepsilon}^{2}|p|^{\sigma_{1}} \\
& \geq \varepsilon^{1-\gamma} \eta_{\varepsilon}^{2}(x)|p|\left(\frac{1}{2}-C \varepsilon^{1+\gamma} \delta^{-1}|p|^{\sigma_{1}-1}\right) \\
& \geq \varepsilon^{1-\gamma} \eta_{\varepsilon}^{2}(x)|p|\left(\frac{1}{2}-C \delta^{-1} \varepsilon^{1+\gamma-\alpha\left(\sigma_{1}-1\right)}\right) .
\end{aligned}
$$

It follows from the assumption $\delta \geq \varepsilon^{\sigma}$ again and (1.40) that

$$
\varepsilon^{1-\gamma} \eta_{\varepsilon}(x)|p|-C \varepsilon^{2} \eta_{\varepsilon}^{2}(x)|p|^{\sigma_{1}} \geq \frac{1}{4} \varepsilon^{1-\gamma} \eta_{\varepsilon}^{2}(x)|p| \geq \frac{1}{6} \varepsilon^{1-\gamma+q} \eta_{\varepsilon}^{2}(x)
$$

when $\varepsilon>0$ is small. In either case, we obtain

$$
Q_{1} \geq-\varepsilon^{2} \delta^{-1} \eta_{\varepsilon}^{2}(x) F\left(x, \nabla \varphi(x), \nabla^{2} \varphi(x)\right)
$$

for any $\varepsilon>0$ small by using (5.13) again. We thus complete the proof of the claim (5.12).

Moreover, it is clear that (3.8) still holds for $Q_{2}$ here. We thus conclude the proof of (5.9) by combining (5.10), (5.12) and (3.8).

Proof of Theorem 5.6. We only show that $\bar{u}^{\sigma}$ is a subsolution. Assume that there exist $\left(x_{0}, t_{0}\right) \in \bar{\Omega} \times(0, \infty)$ and $\phi \in C^{2}(\bar{\Omega} \times[0, \infty))$ such that $\bar{u}-\phi$ attains a unique maximum 
over $\bar{\Omega} \times[0, \infty)$ at $\left(x_{0}, t_{0}\right)$. By definition of $\bar{u}^{\sigma}$, there exist $r>0$ and a sequence $\left(x_{\varepsilon, \delta}, t_{\varepsilon, \delta}\right) \in$ $\bar{\Omega} \times(0, \infty)$ with $\delta \geq \varepsilon^{\sigma}$ such that $\left(x_{\varepsilon, \delta}, t_{\varepsilon, \delta}\right) \rightarrow\left(x_{0}, t_{0}\right)$ as $(\varepsilon, \delta) \rightarrow 0$ and

$$
\left(u^{\varepsilon, \delta}-\phi\right)\left(x_{\varepsilon, \delta}, t_{\varepsilon, \delta}\right) \geq \sup _{B_{r}\left(x_{\varepsilon, \delta}, t-\varepsilon, \delta\right)}\left(u^{\varepsilon}-\phi\right)-\varepsilon^{3} \delta^{2} .
$$

For simplicity of notation below, we use $j$ to denote the index pair $(\varepsilon, \delta)$ with $\delta \geq \varepsilon^{\sigma}$.

It follows that

$$
\phi\left(x_{j}, t_{j}\right) \leq S_{\delta}^{\varepsilon}\left[\varphi_{j}\right]\left(x_{j}\right)+\varepsilon^{3} \delta^{2},
$$

where $\varphi_{j}=\phi\left(\cdot, t_{j}-\varepsilon^{2}\right)$. Applying Lemma 5.9 with $\varphi=\phi_{j}$ and $x=x_{j}$, we deduce that

$$
\begin{aligned}
\phi\left(x_{j}, t_{j}\right)-\phi\left(x_{j}, t_{j}-\varepsilon^{2}\right) \leq- & \varepsilon^{2} \delta^{-1} \eta_{\varepsilon}^{2}\left(x_{j}\right) F\left(x_{j}, \nabla \phi\left(x_{j}, t_{j}-\varepsilon^{2}\right), \nabla^{2} \phi\left(x_{j}, t_{j}-\varepsilon^{2}\right)\right) \\
& -\varepsilon^{2} \zeta_{\varepsilon}\left(x_{j}\right) H\left(x_{j}, \nabla \phi\left(x_{j}, t_{j}-\varepsilon^{2}\right)\right)+o\left(\varepsilon^{2} \delta^{-1} \eta_{\varepsilon}^{2}\left(x_{j}\right)+\varepsilon^{2}\right),
\end{aligned}
$$

which yields that

$$
\begin{aligned}
\varepsilon^{2} \partial_{t} \phi\left(x_{j}, t_{j}\right) \leq- & \varepsilon^{2} \delta^{-1} \eta_{\varepsilon}^{2}\left(x_{j}\right) F\left(x_{j}, \nabla \phi\left(x_{j}, t_{j}-\varepsilon^{2}\right), \nabla^{2} \phi\left(x_{j}, t_{j}-\varepsilon^{2}\right)\right) \\
& -\varepsilon^{2} \zeta_{\varepsilon}\left(x_{j}\right) H\left(x_{j}, \nabla \phi\left(x_{j}, t_{j}-\varepsilon^{2}\right)\right)+o\left(\varepsilon^{2} \delta^{-1} \eta_{\varepsilon}^{2}\left(x_{j}\right)+\varepsilon^{2}\right) .
\end{aligned}
$$

Suppose that $x_{0} \in \Omega$. In this case, we aim to show that

$$
F\left(x_{0}, \nabla \phi\left(x_{0}, t_{0}\right), \nabla^{2} \phi\left(x_{0}, t_{0}\right)\right) \leq 0 .
$$

We may assume that $x_{j} \in \Omega$. It follows from (5.14) that

$$
\begin{aligned}
\eta_{\varepsilon}^{2}\left(x_{j}\right) & F\left(x_{j}, \nabla \phi\left(x_{j}, t_{j}-\varepsilon^{2}\right), \nabla^{2} \phi\left(x_{j}, t_{j}-\varepsilon^{2}\right)\right) \\
& \leq-\delta \partial_{t} \phi\left(x_{j}, t_{j}\right)+\delta \zeta_{\varepsilon}\left(x_{j}\right) H\left(x_{j}, \nabla \phi\left(x_{j}, t_{j}-\varepsilon^{2}\right)\right)+o(1) .
\end{aligned}
$$

Since $\eta_{\varepsilon}\left(x_{j}\right) \rightarrow 1$ as $j \rightarrow 0$, we are led to (5.15) by letting $j \rightarrow 0$.

We next use (5.14) to verify the boundary condition. Suppose that $x_{0} \in \partial \Omega$. By taking a further subsequence, we may discuss the following three cases.

Case 1. If $\delta^{-1} \eta_{\varepsilon}^{2}\left(x_{j}\right) \rightarrow \infty$ as $j \rightarrow 0$, then we may divide (5.14) by $\varepsilon^{2} \delta^{-1} \eta_{\varepsilon}^{2}$ and pass to the limit to get (5.15).

Case 2. If $\delta^{-1} \eta_{\varepsilon}^{2}\left(x_{j}\right) \rightarrow c$ as $j \rightarrow 0$ for some $c \geq 0$, then we have $\eta_{\varepsilon}^{2}\left(x_{j}\right) \rightarrow 0$ and therefore $\zeta_{\varepsilon} \rightarrow 1$ as $j \rightarrow 0$. Dividing (5.14) by $\varepsilon^{2}$ and sending $\varepsilon \rightarrow 0$ yield that

$$
c F\left(x_{0}, \nabla \phi\left(x_{0}, t_{0}\right), \nabla^{2} \phi\left(x_{0}, t_{0}\right)\right)+\partial_{t} \phi\left(x_{0}, t_{0}\right)+H\left(x_{0}, \nabla \phi\left(x_{0}, t_{0}\right)\right) \leq 0 .
$$

Hence, combining both cases above, we are led to

$$
\min \left\{F\left(x_{0}, \nabla \phi\left(x_{0}, t_{0}\right), \nabla^{2} \phi\left(x_{0}, t_{0}\right)\right), \partial_{t} \phi\left(x_{0}, t_{0}\right)+H\left(x_{0}, \nabla \phi\left(x_{0}, t_{0}\right)\right)\right\} \leq 0,
$$

which completes the proof.

5.3.2. Game convergence. Suppose that (F3) holds. As pointed out in Remark 3.4, the ranges for $\alpha, \beta, \gamma$ in this case become simpler; see (3.11). For convergence of $u^{\varepsilon, \delta}$ with $\delta \geq \varepsilon^{\sigma}$, the choice of $\sigma$ becomes also simpler. Indeed, as $\sigma_{1}=\sigma_{2}=1$, the conditions (1.38)-(1.41) reduce to only $\sigma<2 \gamma$.

Theorem 5.10 (Game convergence for elliptic equations with dynamic boundary conditions). Assume that $\Omega \subset \mathbb{R}^{n}$ is a bounded $C^{2,1}$ domain. Assume that (F3), (F4), (F5), (H1), (H2), (B1) and (B2) hold. Assume that (1.20)-(1.24) hold. Assume that $u_{0}$ is Lipschitz in $\bar{\Omega}$. Let $\varepsilon, \delta>0$ and $u^{\varepsilon, \delta}$ be the game value in (1.16) but with running cost $F$ in (1.15) replaced by $F / \delta$. Then $u^{\varepsilon, \delta} \rightarrow U$ locally uniformly in $\bar{\Omega} \times(0, \infty)$ as $(\varepsilon, \delta) \rightarrow 0$ 
provided that $\delta \geq \varepsilon^{\sigma}$ with $\sigma<2 \gamma$, where $U$ is the unique solution of (DBP3) with the initial value $u_{\infty}$ being the unique solution of (DP2).

Remark 5.11. The convergence of $u^{\varepsilon, \delta}$ does not apply to the initial moment. This is certainly due to an instant jump from $u_{0}$ to $u_{\infty}$.

Since in Section 3 we have shown the locally uniform convergence $u^{\varepsilon, \delta}$ to $u_{\delta}$ as $\varepsilon \rightarrow 0$, Theorem 5.10 immediately implies the following result.

Corollary 5.12 (Fast evolution behavior). Assume that $\Omega \subset \mathbb{R}^{n}$ is a bounded $C^{2,1}$ domain satisfying (H1) and (H2). Assume that (F3), (F4), (F5), (B1) and (B2) hold. Assume that $u_{0}$ is Lipschitz in $\bar{\Omega}$. Let $u_{\delta}$ be the unique solution of (DBP2). Then $u_{\delta} \rightarrow U$ locally uniformly in $\bar{\Omega} \times(0, \infty)$ as $\delta \rightarrow 0$, where $U$ is the unique solution of (DBP3) with $u_{\infty}$ being the unique solution of (DP2).

Let us show Theorem 5.10 by using Theorem 5.6. Since under the assumption (F3) we can assume $\sigma<2 \gamma$ in place of (1.38)-(1.41), the definition of relaxed half limits as in (5.6) can be reduced to

$$
\begin{array}{r}
\bar{u}^{\sigma}(x, t)=\lim _{r \rightarrow 0} \sup \left\{u^{\varepsilon, \delta}(y, s):(y, s) \in \bar{\Omega} \times[0, \infty),|x-y|+|t-s|+\varepsilon+\delta \leq r,\right. \\
\left.\delta \geq \varepsilon^{\sigma} \text { with } 0<\sigma<2 \gamma\right\}, \\
\underline{u}_{\sigma}(x, t)=\lim _{r \rightarrow 0} \inf \left\{u^{\varepsilon, \delta}(y, s):(y, s) \in \bar{\Omega} \times[0, \infty),|x-y|+|t-s|+\varepsilon+\delta \leq r,\right. \\
\left.\delta \geq \varepsilon^{\sigma} \text { with } 0<\sigma<2 \gamma\right\} .
\end{array}
$$

We prove that $\bar{u}^{\sigma}$ and $\underline{u}_{\sigma}$ above essentially satisfies the initial value (1.35).

Proposition 5.13 (Initial verification). Assume that $\Omega \subset \mathbb{R}^{n}$ is a bounded $C^{2}$ domain satisfying (H1) and (H2). Assume that (F3), (F4), (F5), (B1) and (B2) hold. Let $u_{0}$ be Lipschitz in $\bar{\Omega}$. Let $\varepsilon, \delta>0$ and $u^{\varepsilon, \delta}$ be the game value in (1.16) but with running cost $F$ in (1.15) replaced by $F / \delta$. Let $\bar{u}^{\sigma}$ and $\underline{u}_{\sigma}$ be defined as in (5.16). Then $\bar{u}^{\sigma}(x, t)$ and $\underline{u}_{\sigma}(x, t)$ converge to $u_{\infty}(x)$ uniformly for all $x \in \bar{\Omega}$ as $t \rightarrow 0$.

Proof. By (5.8), we have for any $\tau>0$ and any $t>0$

$$
\bar{u}^{\sigma}(\cdot, t) \leq u_{0}+\tau+M_{\tau} t+\omega(t) \quad \text { on } \partial \Omega .
$$

A symmetric argument yields

$$
\underline{u}_{\sigma}(\cdot, t) \geq u_{0}-\tau-M_{\tau} t+\omega(t) \quad \text { on } \partial \Omega .
$$

Moreover, by Theorem 5.6, we see that $\bar{u}^{\sigma}$ and $\underline{u}_{\sigma}$, as functions of $(x, t)$ in $\Omega \times(0, \infty)$, are respectively a subsolution and a supersolution of (1.34), which implies that $\bar{u}^{\sigma}(\cdot, t)$ and $\underline{u}_{\sigma}(\cdot, t)$ are respectively a subsolution and a supersolution of (1.34) for all $t>0$.

Indeed, suppose that for any fixed $t_{0}>0$, there is a function $\varphi \in C^{2}(\Omega)$ such that $\bar{u}^{\sigma}\left(\cdot, t_{0}\right)-\varphi$ attains a strict maximum at $x_{0} \in \Omega$. Then, due to the boundedness of $\bar{u}^{\sigma}$ and $\bar{\Omega}$, for any $m>0$ large,

$$
(x, t) \mapsto \bar{u}^{\sigma}(x, t)-\varphi(x)-m\left|t-t_{0}\right|^{2}
$$


attains a local maximum at $\left(x_{m}, t_{m}\right) \in \bar{\Omega} \times(0, \infty)$. We then have

$$
\bar{u}^{\sigma}\left(x_{m}, t_{m}\right)-\varphi\left(x_{m}\right)-m\left|t_{m}-t_{0}\right|^{2} \geq \bar{u}^{\sigma}\left(x_{0}, t_{0}\right)-\varphi\left(x_{0}\right),
$$

which yields $t_{m} \rightarrow t_{0}$ as $m \rightarrow \infty$. This further implies that $x_{m} \rightarrow x_{0}$ as $m \rightarrow \infty$. If this is not the case, we may take a subsequence, still indexed by $m$ for convenience, such that $x_{m} \rightarrow \hat{x} \in \bar{\Omega}$ as $m \rightarrow \infty$. Then by (5.18), we have

$$
\bar{u}^{\sigma}\left(\hat{x}, t_{0}\right)-\varphi(\hat{x}) \geq \limsup _{m \rightarrow \infty}\left(\bar{u}^{\sigma}\left(x_{m}, t_{m}\right)-\varphi\left(x_{m}\right)\right) \geq \bar{u}^{\sigma}\left(x_{0}, t_{0}\right)-\varphi\left(x_{0}\right),
$$

which is a contradiction. Hence we have $x_{m} \in \Omega$.

By the definition of viscosity subsolutions of (1.34), we thus obtain

$$
F\left(x_{m}, \nabla \varphi\left(x_{m}\right), \nabla^{2} \varphi\left(x_{m}\right)\right) \leq 0,
$$

and therefore

$$
F\left(x_{0}, \nabla \varphi\left(x_{0}\right), \nabla^{2} \varphi\left(x_{0}\right)\right) \leq 0
$$

by letting $m \rightarrow \infty$. The same argument applies to $\underline{u}_{\sigma}$ as well.

Recall from Proposition 5.4 that $u_{\infty}$ the unique solution of (DP2). We can use the comparison principle for (DP2) (Theorem 5.2) to get

$$
\bar{u}^{\sigma}(x, t) \leq u_{\infty}(x)+\tau+M_{\tau} t+\omega(t)
$$

for all $(x, t) \in \bar{\Omega} \times(0, \infty)$ and any $\tau>0$. A symmetric argument yields

$$
\underline{u}_{\sigma}(x, t) \leq u_{\infty}(x)-\tau-M_{\tau} t+\omega(t)
$$

for all $(x, t) \in \bar{\Omega} \times(0, \infty)$ and any $\tau>0$. Noticing that $\underline{u}_{\sigma} \leq \bar{u}$, we have

$$
\left|\bar{u}^{\sigma}(x, t)-u_{\infty}(x)\right| \leq \tau+M t+\omega(t) .
$$

This means that for any $\lambda>0$, we can let

$$
\left|\bar{u}^{\sigma}(x, t)-u_{\infty}\right| \leq \lambda
$$

by choosing $\tau \leq \lambda / 2$ first and then letting $t>0$ small such that $M t+o(t) \leq \lambda / 2$. This shows that $\bar{u}^{\sigma}(\cdot, t)$ uniformly converges to $u_{\infty}$ in $\bar{\Omega}$ as $t \rightarrow 0$. A similar result holds also for $\underline{u}_{\sigma}$.

We are now in a position to prove Theorem 5.10.

Proof of Theorem 5.10. By Theorem 5.6, we see that $\bar{u}^{\sigma}$ and $\underline{u}_{\sigma}$ are respectively a subsolution and a supersolution of (1.34) satisfying the dynamic boundary condition (1.2). Moreover, Proposition 5.13 allows us to redefine the initial values of $\bar{u}^{\sigma}$ and $\underline{u}_{\sigma}$ to be

$$
\bar{u}^{\sigma}(\cdot, 0)=\underline{u}_{\sigma}(\cdot, 0)=u_{\infty} \quad \text { in } \bar{\Omega} .
$$

The convergence of $u^{\varepsilon, \delta}$ to the unique solution of (DBP3) is therefore a consequence of Theorem 2.5. 


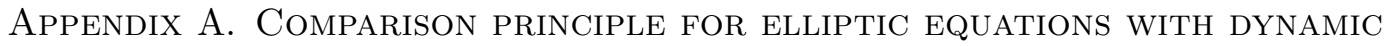
BOUNDARY CONDITIONS

We below give a proof of Theorem 5.5 based on an adaptation of the arguments by Barles [8]. Let us prepare several preliminary results that will be used in the proof of Theorem 5.5. Given $\lambda>0$ small, $(x, b, p) \in\left(\bar{\Omega} \backslash \Omega_{\lambda}\right) \times \mathbb{R} \times \mathbb{R}^{n}$, we choose a real number $C(x, b, p)$ such that

$$
b+H(x, p+C(x, b, p) \nu(x))=0 .
$$

Using the method in $[7,8]$, we can extend $C$ to a function defined in $\mathbb{R}^{n} \times \mathbb{R} \times \mathbb{R}^{n}$ and approximate this function by a family of smooth functions $\left\{C_{\eta}\right\}_{\eta>0}$.

Proposition A.1. Assume that $\Omega$ is a bounded $C^{2,1}$ domain. Assume (B1) and (B2). Let $H$ be given by (1.8). Let $C:\left(\bar{\Omega} \backslash \Omega_{\lambda}\right) \times \mathbb{R} \times \mathbb{R}^{n} \rightarrow \mathbb{R}$ be the function satisfying (A.1). Then for each $\eta>0$ there exists a smooth function $C_{\eta}: \mathbb{R}^{n} \times \mathbb{R} \times \mathbb{R}^{n} \rightarrow \mathbb{R}$ such that for any $R>0$,

$$
\left|C_{\eta}(x, b, p)-C(x, b, p)\right| \leq K_{0}(\eta+|\langle p, \nu(x)\rangle|)
$$

holds for all $(x, b, p) \in\left(\bar{\Omega} \backslash \Omega_{\lambda}\right) \times[-R, R] \times \mathbb{R}^{n}$ with $K_{0}>0$ independent of $\eta, x, b, p$, and

$$
\begin{aligned}
\left|C_{\eta}(x, 0,0)\right| & \leq K, \\
\left|\nabla_{x} C_{\eta}(x, b, p)\right| & \leq K(1+|p|), \\
\left|\frac{\partial}{\partial b} C_{\eta}(x, b, p)\right| & \leq K, \\
\left|\nabla_{p} C_{\eta}(x, b, p)\right| & \leq K, \\
\left|\nabla_{x}^{2} C_{\eta}(x, b, p)\right| & \leq K(1+|p|)^{2}(\eta+|\langle p, \nu(x)\rangle|)^{-1}, \\
\left|\nabla_{x p}^{2} C_{\eta}(x, b, p)\right| & \leq K(1+|p|)(\eta+|\langle p, \nu(x)\rangle|)^{-1}, \\
\left|\nabla_{p}^{2} C_{\eta}(x, b, p)\right| & \leq K(\eta+|\langle p, \nu(x)\rangle|)^{-1}
\end{aligned}
$$

hold for all $(x, b, p) \in \mathbb{R}^{n} \times[-R, R] \times \mathbb{R}^{n}$, where $K>0$ depends on $\eta$ and $R$.

We omit precise description on how to construct $C_{\eta}$, which can be found in $[8$, Proof of Lemma 5.1, Step 1]. Note that the boundary condition considered in [8, Lemma 5.1] does not include the time derivative $u_{t}$ (represented by $b$ above) but depends on the unknown function $u$ instead. We actually can build $C_{\eta}$ here by letting $b$ take the role of $u$ in the regularization process there and all estimates still hold for our purpose.

As a result, we obtain the following.

Proposition A.2. Suppose that all assumptions in Lemma A.1 hold. Then $C_{\eta}$ in Lemma A.1 satisfies

$$
\left|b+H\left(x, p+C_{\eta}(x, b, p) \nu(x)\right)\right| \leq K_{0}(\eta+|\langle p, \nu(x)\rangle|)
$$

and

$$
\left|C_{\eta}(x, b, p)\right| \leq K(1+|b|+|p|)
$$

for all $(x, b, p) \in \mathbb{R}^{n} \times[-R, R] \times \mathbb{R}^{n}$ and $\eta>0$.

Proof. Recall that (B1) implies the Lipschitz regularity (1.10) of $H$. Using (A.1), (1.10) and (A.2), we have

$$
\begin{aligned}
\left|b+H\left(x, p+C_{\eta}(x, b, p) \nu(x)\right)\right| & =\left|H(x, p+C(x, b, p) \nu(x))-H\left(x, p+C_{\eta}(x, b, p) \nu(x)\right)\right| \\
& \leq L\left|C_{\eta}(x, b, p)-C(x, b, p)\right| \leq L K_{0}(\eta+|\langle p, \nu(x)\rangle|) .
\end{aligned}
$$


We thus obtain (A.10) by resetting $L K_{0}=K_{0}$. Next, it follows from (A.3), (A.5) and (A.6) that

$$
\left|C_{\eta}(x, b, p)\right| \leq\left|C_{\eta}(x, 0,0)\right|+\left|C_{\eta}(x, b, p)-C_{\eta}(x, 0,0)\right| \leq K+K(|b|+|p|),
$$

which shows (A.11).

Lemma A.3 ([7, page $99(20)])$. Assume $\Omega$ is a bounded $C^{2}$ domain. Let $d(x)$ denote the signed distance from $x \in \bar{\Omega}$ to $\partial \Omega$. Then for all $x, y \in \bar{\Omega}$

$$
d(x)-d(y)=\left\langle(y-x), \nu\left(\frac{x+y}{2}\right)\right\rangle+O\left(|x-y|^{2}\right) .
$$

In particular, if $x \in \partial \Omega$, then

$$
\left\langle(x-y), \nu\left(\frac{x+y}{2}\right)\right\rangle=d(y)+O\left(|x-y|^{2}\right) .
$$

Proof. By the regularity of $d$ we have

$$
d(x)-d(y)=\langle\nabla d(y), x-y\rangle+O\left(|x-y|^{2}\right)
$$

and

$$
\nabla d(y)=\nabla d\left(\frac{x+y}{2}\right)+O(|x-y|)=-\nu\left(\frac{x+y}{2}\right)+O(|x-y|) .
$$

These two equations yield (A.12). Also, (A.13) is an immediate consequence of (A.12) since $d(x)=0$.

Proof of Theorem 5.5. Suppose by contradiction that $M:=u\left(x_{0}, t_{0}\right)-v\left(x_{0}, t_{0}\right)>0$ for some $\left(x_{0}, t_{0}\right) \in \bar{\Omega} \times(0, \infty)$. Fix $T>t_{0}$.

1. Doubling variables. Define an upper semicontinuous function $\Phi:(\bar{\Omega} \times[0, T))^{2} \rightarrow \mathbb{R}$ by

$$
\Phi(x, t, y, s):=u(x, t)-v(y, s)-\psi(x, t, y, s)
$$

where

$$
\begin{aligned}
\psi(x, t, y, s):= & \frac{|x-y|^{2}}{\varepsilon^{2}}+\frac{(t-s)^{2}}{\alpha^{2}}+\frac{A(d(x)-d(y))^{2}}{\varepsilon^{2}}+\frac{\delta}{T-t}+\frac{\delta}{T-s} \\
& -C_{\eta}\left(\frac{x+y}{2}, \frac{2(t-s)}{\alpha^{2}}, \frac{2(x-y)}{\varepsilon^{2}}\right)(d(x)-d(y)) .
\end{aligned}
$$

Here $\delta$ is taken to be $4 M /\left(T-t_{0}\right)$ and $A>0$ is a fixed constant to be determined later. We may let $\varepsilon, \alpha>0$ and $\eta>0$ small such that $\Phi$ attains a maximum over $(\bar{\Omega} \times[0, T))^{2}$ at a point $Z_{\varepsilon}=\left(x_{\varepsilon}, t_{\varepsilon}, y_{\varepsilon}, s_{\varepsilon}\right)$.

A standard viscosity argument then yields that

- There exists $(\bar{x}, \bar{t}, \bar{x}, \bar{s}) \in(\bar{\Omega} \times[0, T))^{2}$ such that

$$
Z_{\varepsilon}=\left(x_{\varepsilon}, t_{\varepsilon}, y_{\varepsilon}, s_{\varepsilon}\right) \rightarrow(\bar{x}, \bar{t}, \bar{x}, \bar{s})
$$

as $\varepsilon \rightarrow 0$ by taking a subsequence; below we still index the subsequence by $\varepsilon$ for convenience.

- $x_{\varepsilon}, y_{\varepsilon}$ are close to each other in the sense that

$$
\frac{\left|x_{\varepsilon}-y_{\varepsilon}\right|^{2}}{\varepsilon^{2}} \rightarrow 0 \quad \text { as } \varepsilon \rightarrow 0
$$


- $t_{\varepsilon} \neq 0$ and $s_{\varepsilon} \neq 0$ and

$$
\left|t_{\varepsilon}-s_{\varepsilon}\right| \leq \alpha
$$

for all $\varepsilon, \alpha$ small enough. We fix such $\alpha>0$.

Set

$$
Q:=\left(\frac{x+y}{2}, \frac{2(t-s)}{\alpha^{2}}, \frac{2(x-y)}{\varepsilon^{2}}\right) .
$$

As (A.15) essentially gives a bound $2 / \alpha$ for the variable $b$ of $C_{\eta}$, below we will apply the estimates (A.3)-(A.11) with $R=2 / \alpha$. For later use, we differentiate $\psi$ to obtain

$$
\begin{aligned}
& \partial_{t} \psi(x, t, y, s)=\frac{2(t-s)}{\alpha^{2}}+\frac{\delta}{(T-t)^{2}}-\frac{\partial}{\partial b} C_{\eta}(Q) \frac{2(d(x)-d(y))}{\alpha^{2}}, \\
& \nabla_{x} \psi(x, t, y, s)= \frac{2(x-y)}{\varepsilon^{2}}+\frac{2 A(d(x)-d(y))}{\varepsilon^{2}} \nabla d(x) \\
&-\left(\frac{1}{2} \nabla_{x} C_{\eta}(Q)+\frac{2}{\varepsilon^{2}} \nabla_{p} C_{\eta}(Q)\right)(d(x)-d(y))-C_{\eta}(Q) \nabla d(x), \\
& \nabla_{y} \psi(x, t, y, s)=-\frac{2(x-y)}{\varepsilon^{2}}-\frac{2 A(d(x)-d(y))}{\varepsilon^{2}} \nabla d(y) \\
&-\left(\frac{1}{2} \nabla_{y} C_{\eta}(Q)-\frac{2}{\varepsilon^{2}} \nabla_{p} C_{\eta}(Q)\right)(d(x)-d(y))+C_{\eta}(Q) \nabla d(y) .
\end{aligned}
$$

2. Violation of the boundary conditions. Let us show that

$$
\partial_{t} \psi\left(Z_{\varepsilon}\right)+H\left(x_{\varepsilon}, \nabla_{x} \psi\left(Z_{\varepsilon}\right)\right)>0 \quad \text { if } x_{\varepsilon} \in \partial \Omega
$$

and

$$
-\partial_{s} \psi\left(Z_{\varepsilon}\right)+H\left(y_{\varepsilon},-\nabla_{y} \psi\left(Z_{\varepsilon}\right)\right)<0 \quad \text { if } y_{\varepsilon} \in \partial \Omega
$$

for $\varepsilon>0$ small enough. Here we only give the proof of (A.19) since the same argument works for (A.20). If $x_{\varepsilon} \in \partial \Omega$, since $d\left(x_{\varepsilon}\right)=0$ and $\nabla d(x)=-\nu(x)$, by (A.16) and (A.17) we have

$$
\begin{aligned}
\partial_{t} \psi\left(Z_{\varepsilon}\right)= & \frac{2\left(t_{\varepsilon}-s_{\varepsilon}\right)}{\alpha^{2}}+\frac{\delta}{\left(T-t_{\varepsilon}\right)^{2}}+\frac{2 d\left(y_{\varepsilon}\right)}{\alpha^{2}} \frac{\partial C_{\eta}}{\partial b}\left(Q_{\varepsilon}\right) \\
\nabla_{x} \psi\left(Z_{\varepsilon}\right)= & \frac{2\left(x_{\varepsilon}-y_{\varepsilon}\right)}{\varepsilon^{2}}+\frac{2 A d\left(y_{\varepsilon}\right)}{\varepsilon^{2}} \nu\left(x_{\varepsilon}\right) \\
& +\left(\frac{1}{2} \nabla_{x} C_{\eta}\left(Q_{\varepsilon}\right)+\frac{2}{\varepsilon^{2}} \nabla_{p} C_{\eta}\left(Q_{\varepsilon}\right)\right) d\left(y_{\varepsilon}\right)+C_{\eta}\left(Q_{\varepsilon}\right) \nu\left(x_{\varepsilon}\right),
\end{aligned}
$$

where

$$
Q_{\varepsilon}:=\left(\frac{x_{\varepsilon}+y_{\varepsilon}}{2}, \frac{2\left(t_{\varepsilon}-s_{\varepsilon}\right)}{\alpha^{2}}, \frac{2\left(x_{\varepsilon}-y_{\varepsilon}\right)}{\varepsilon^{2}}\right)
$$

Let us now give an estimate for each term on the left hand side of (A.19) for $\varepsilon>0$ small.

By (A.5), we get

$$
\left|\frac{2 d\left(y_{\varepsilon}\right)}{\alpha^{2}} \frac{\partial}{\partial b} C_{\eta}\left(Q_{\varepsilon}\right)\right| \leq \frac{2 K\left|x_{\varepsilon}-y_{\varepsilon}\right|}{\alpha^{2}}=o(1)
$$

for $\varepsilon>0$ small. Here and in the sequel, the constant implied in the error term $o(1)$ also depends on $\alpha, A, \delta, \eta$. 
We next use (A.10) and (A.13) to deduce that

$$
\begin{aligned}
& \frac{2\left(t_{\varepsilon}-s_{\varepsilon}\right)}{\alpha^{2}}+H\left(\frac{x_{\varepsilon}+y_{\varepsilon}}{2}, \frac{2\left(x_{\varepsilon}-y_{\varepsilon}\right)}{\varepsilon^{2}}+C_{\eta}\left(Q_{\varepsilon}\right) \nu\left(\frac{x_{\varepsilon}+y_{\varepsilon}}{2}\right)\right) \\
& \geq-K_{0}\left(\eta+\left|\left\langle\frac{2\left(x_{\varepsilon}-y_{\varepsilon}\right)}{\varepsilon^{2}}, \nu\left(\frac{x_{\varepsilon}+y_{\varepsilon}}{2}\right)\right\rangle\right|\right) \\
& \geq-K_{0}\left(\eta+\frac{2}{\varepsilon^{2}}\left(d\left(y_{\varepsilon}\right)+O\left(\left|x_{\varepsilon}-y_{\varepsilon}\right|^{2}\right)\right)\right)=-K_{0} \eta-2 K_{0} \frac{d\left(y_{\varepsilon}\right)}{\varepsilon^{2}}+o(1) .
\end{aligned}
$$

Moreover, using the Lipschitz regularity of $H$ as in (1.10) implied by (B1), we see that

$$
\begin{aligned}
H\left(x_{\varepsilon}, \nabla_{x} \psi\left(Z_{\varepsilon}\right)\right) \geq H & \left(x_{\varepsilon}, \frac{2\left(x_{\varepsilon}-y_{\varepsilon}\right)}{\varepsilon^{2}}+\frac{2 A d\left(y_{\varepsilon}\right)}{\varepsilon^{2}} \nu\left(x_{\varepsilon}\right)+C_{\eta}\left(Q_{\varepsilon}\right) \nu\left(x_{\varepsilon}\right)\right) \\
& -L_{H}\left|\frac{1}{2} \nabla_{x} C_{\eta}\left(Q_{\varepsilon}\right)+\frac{2}{\varepsilon^{2}} \nabla_{p} C_{\eta}\left(Q_{\varepsilon}\right)\right| d\left(y_{\varepsilon}\right) .
\end{aligned}
$$

It follows by (1.11) (due to (B2)) that

$$
\begin{aligned}
H\left(x_{\varepsilon}, \nabla_{x} \psi\left(Z_{\varepsilon}\right)\right) \geq & H\left(x_{\varepsilon}, \frac{2\left(x_{\varepsilon}-y_{\varepsilon}\right)}{\varepsilon^{2}}+C_{\eta}\left(Q_{\varepsilon}\right) \nu\left(x_{\varepsilon}\right)\right)+\frac{2 \rho A d\left(y_{\varepsilon}\right)}{\varepsilon^{2}} \\
& -L_{H}\left|\frac{1}{2} \nabla_{x} C_{\eta}\left(Q_{\varepsilon}\right)+\frac{2}{\varepsilon^{2}} \nabla_{p} C_{\eta}\left(Q_{\varepsilon}\right)\right| d\left(y_{\varepsilon}\right) .
\end{aligned}
$$

Our goal is to estimate from below the difference

$$
H\left(x_{\varepsilon}, \nabla_{x} \psi\left(Z_{\varepsilon}\right)\right)-H\left(\frac{x_{\varepsilon}+y_{\varepsilon}}{2}, \frac{2\left(x_{\varepsilon}-y_{\varepsilon}\right)}{\varepsilon^{2}}+C_{\eta}\left(Q_{\varepsilon}\right) \nu\left(\frac{x_{\varepsilon}+y_{\varepsilon}}{2}\right)\right) .
$$

To this end, adopting (A.4) and (A.6), we see that the last term on the right hand side of (A.24) satisfies

$$
\begin{aligned}
& L_{H}\left|\frac{1}{2} \nabla_{x} C_{\eta}\left(Q_{\varepsilon}\right)+\frac{2}{\varepsilon^{2}} \nabla_{p} C_{\eta}\left(Q_{\varepsilon}\right)\right| d\left(y_{\varepsilon}\right) \\
& \leq \frac{L_{H}}{2}\left|\nabla_{x} C_{\eta}\left(Q_{\varepsilon}\right)\right| d\left(y_{\varepsilon}\right)+2 L_{H}\left|\nabla_{p} C_{\eta}\left(Q_{\varepsilon}\right)\right| \frac{d\left(y_{\varepsilon}\right)}{\varepsilon^{2}} \\
& \leq \frac{L_{H} K}{2}\left(1+\frac{2\left|x_{\varepsilon}-y_{\varepsilon}\right|}{\varepsilon^{2}}\right)\left|x_{\varepsilon}-y_{\varepsilon}\right|+2 L_{H} K \frac{d\left(y_{\varepsilon}\right)}{\varepsilon^{2}}=o(1)+2 L_{H} K \frac{d\left(y_{\varepsilon}\right)}{\varepsilon^{2}} .
\end{aligned}
$$

On the other hand, in view of (1.10) we deduce that

$$
\begin{aligned}
& H\left(\frac{x_{\varepsilon}+y_{\varepsilon}}{2}, q_{\varepsilon, 2}\right)-H\left(x_{\varepsilon}, q_{\varepsilon, 1}\right) \\
& \leq L_{H}\left(1+\left|q_{\varepsilon, 1}\right|+\left|q_{\varepsilon, 2}\right|\right)\left|\frac{x_{\varepsilon}-y_{\varepsilon}}{2}\right|+L_{H}\left|C_{\eta}\left(Q_{\varepsilon}\right)\right|\left|\nu\left(x_{\varepsilon}\right)-\nu\left(\frac{x_{\varepsilon}+y_{\varepsilon}}{2}\right)\right|,
\end{aligned}
$$

where

$$
\begin{gathered}
q_{\varepsilon, 1}:=\frac{2\left(x_{\varepsilon}-y_{\varepsilon}\right)}{\varepsilon^{2}}+C_{\eta}\left(Q_{\varepsilon}\right) \nu\left(x_{\varepsilon}\right), \\
q_{\varepsilon, 2}:=\frac{2\left(x_{\varepsilon}-y_{\varepsilon}\right)}{\varepsilon^{2}}+C_{\eta}\left(Q_{\varepsilon}\right) \cdot \nu\left(\frac{x_{\varepsilon}+y_{\varepsilon}}{2}\right) .
\end{gathered}
$$


Thanks to (A.15) and (A.11), the right hand side of (A.26) can be estimated as follows:

$$
\begin{gathered}
L_{H}\left(1+\left|q_{\varepsilon, 1}\right|+\left|q_{\varepsilon, 2}\right|\right)\left|\frac{x_{\varepsilon}-y_{\varepsilon}}{2}\right| \leq L_{H}\left(1+\frac{4\left|x_{\varepsilon}-y_{\varepsilon}\right|}{\varepsilon^{2}}+2\left|C_{\eta}\left(Q_{\varepsilon}\right)\right|\right) \frac{\left|x_{\varepsilon}-y_{\varepsilon}\right|}{2} \\
\leq L_{H}\left(1+\frac{4\left|x_{\varepsilon}-y_{\varepsilon}\right|}{\varepsilon^{2}}+2 K\left(1+\frac{2}{\alpha}+\frac{2\left|x_{\varepsilon}-y_{\varepsilon}\right|}{\varepsilon^{2}}\right)\right) \frac{\left|x_{\varepsilon}-y_{\varepsilon}\right|}{2}=o(1), \\
L_{H}\left|C_{\eta}\left(Q_{\varepsilon}\right)\right|\left|\nu\left(x_{\varepsilon}\right)-\nu\left(\frac{x_{\varepsilon}+y_{\varepsilon}}{2}\right)\right| \leq L_{H} K\left(1+\frac{2}{\alpha}+\frac{2\left|x_{\varepsilon}-y_{\varepsilon}\right|}{\varepsilon^{2}}\right) O\left(\left|x_{\varepsilon}-y_{\varepsilon}\right|\right)=o(1) .
\end{gathered}
$$

Hence, we have

$$
H\left(x_{\varepsilon}, q_{\varepsilon, 1}\right)-H\left(\frac{x_{\varepsilon}+y_{\varepsilon}}{2}, q_{\varepsilon, 2}\right) \geq o(1) .
$$

Combining (A.24), (A.25) and (A.27), we are led to

$$
\begin{aligned}
H\left(x_{\varepsilon}, \nabla_{x} \psi\left(Z_{\varepsilon}\right)\right)- & H\left(\frac{x_{\varepsilon}+y_{\varepsilon}}{2}, \frac{2\left(x_{\varepsilon}-y_{\varepsilon}\right)}{\varepsilon^{2}}+C_{\eta}\left(Q_{\varepsilon}\right) \nu\left(\frac{x_{\varepsilon}+y_{\varepsilon}}{2}\right)\right) \\
\geq & \frac{2 \rho A d\left(y_{\varepsilon}\right)}{\varepsilon^{2}}-2 L_{H} K \frac{d\left(y_{\varepsilon}\right)}{\varepsilon^{2}}+o(1) .
\end{aligned}
$$

We finally obtain the estimate

$$
\begin{aligned}
& \partial_{t} \psi\left(Z_{\varepsilon}\right)+H\left(x_{\varepsilon}, \nabla_{x} \psi\left(Z_{\varepsilon}\right)\right) \\
& \geq \frac{\delta}{\left(T-t_{\varepsilon}\right)^{2}}-K_{0} \eta+\frac{d\left(y_{\varepsilon}\right)}{\varepsilon^{2}}\left(2 \rho A-2 K_{0}-2 L_{H} K\right)-o(1) .
\end{aligned}
$$

This shows that (A.19) holds for $\varepsilon>0$ small enough when $\eta$ and $A$ are chosen to satisfy

$$
\eta \leq \frac{\delta}{2 K_{0} T^{2}}, \quad A \geq \frac{K_{0}+L_{H} K}{\rho} .
$$

3. Further estimates for the derivatives of $\psi$. We first consider $\nabla_{x} \psi\left(Z_{\varepsilon}\right)+\nabla_{y} \psi\left(Z_{\varepsilon}\right)$, which is estimated using (A.17) and (A.18) as follows:

$$
\begin{aligned}
& \left|\nabla_{x} \psi\left(Z_{\varepsilon}\right)+\nabla_{y} \psi\left(Z_{\varepsilon}\right)\right| \\
& \leq\left|\frac{2 A\left(d\left(x_{\varepsilon}\right)-d\left(y_{\varepsilon}\right)\right)-C_{\eta}\left(Q_{\varepsilon}\right)}{\varepsilon^{2}}\left(\nabla d\left(x_{\varepsilon}\right)-\nabla d\left(y_{\varepsilon}\right)\right)-\nabla_{x} C_{\eta}\left(Q_{\varepsilon}\right)\left(d\left(x_{\varepsilon}\right)-d\left(y_{\varepsilon}\right)\right)\right| \\
& \leq \frac{2 A\left|d\left(x_{\varepsilon}\right)-d\left(y_{\varepsilon}\right)\right|+\left|C_{\eta}\left(Q_{\varepsilon}\right)\right|}{\varepsilon^{2}}\left|\nabla d\left(x_{\varepsilon}\right)-\nabla d\left(y_{\varepsilon}\right)\right|+\left|\nabla_{x} C_{\eta}\left(Q_{\varepsilon}\right)\right|\left|d\left(x_{\varepsilon}\right)-d\left(y_{\varepsilon}\right)\right| \\
& \leq\left(K+\frac{(2 A+2 K)\left|x_{\varepsilon}-y_{\varepsilon}\right|}{\varepsilon^{2}}\right) O\left(\left|x_{\varepsilon}-y_{\varepsilon}\right|\right)+K\left(1+\frac{2\left|x_{\varepsilon}-y_{\varepsilon}\right|}{\varepsilon^{2}}\right)\left|x_{\varepsilon}-y_{\varepsilon}\right| .
\end{aligned}
$$

We thus have

$$
\left|\nabla_{x} \psi\left(Z_{\varepsilon}\right)+\nabla_{y} \psi\left(Z_{\varepsilon}\right)\right|=o(1) .
$$

Utilizing (A.17) again, we observe that

$$
\begin{aligned}
\left|\nabla_{x} \psi\left(Z_{\varepsilon}\right)\right| \leq & \frac{2\left|x_{\varepsilon}-y_{\varepsilon}\right|}{\varepsilon^{2}}+\frac{2 A\left|d\left(x_{\varepsilon}\right)-d\left(y_{\varepsilon}\right)\right|}{\varepsilon^{2}}+\left|C_{\eta}\left(Q_{\varepsilon}\right)\right| \\
& +\left(\frac{1}{2}\left|\nabla_{x} C_{\eta}\left(Q_{\varepsilon}\right)\right|+\frac{2}{\varepsilon^{2}}\left|\nabla_{p} C_{\eta}\left(Q_{\varepsilon}\right)\right|\right)\left|d\left(x_{\varepsilon}\right)-d\left(y_{\varepsilon}\right)\right| \\
\leq & \frac{2\left|x_{\varepsilon}-y_{\varepsilon}\right|}{\varepsilon^{2}}+\frac{2 A\left|x_{\varepsilon}-y_{\varepsilon}\right|}{\varepsilon^{2}}+K\left(1+\frac{2\left|x_{\varepsilon}-y_{\varepsilon}\right|}{\varepsilon^{2}}\right) \\
& +\left(\frac{1}{2} K\left(1+\frac{2\left|x_{\varepsilon}-y_{\varepsilon}\right|}{\varepsilon^{2}}\right)+\frac{2}{\varepsilon^{2}} K\right)\left|x_{\varepsilon}-y_{\varepsilon}\right|,
\end{aligned}
$$


which implies that

$$
\left|\nabla_{x} \psi\left(Z_{\varepsilon}\right)\right|\left|x_{\varepsilon}-y_{\varepsilon}\right|=o(1)
$$

when $\varepsilon>0$ is sufficiently small. In a similar way, we have

$$
\left|\nabla_{y} \psi\left(Z_{\varepsilon}\right)\right|\left|x_{\varepsilon}-y_{\varepsilon}\right|=o(1) .
$$

The second derivative of $\psi$ is more involved to handle. In the same manner as in $[7$, page 99,100$]$, we can obtain

$$
\nabla^{2} \psi\left(Z_{\varepsilon}\right) \leq O\left(\frac{1}{\varepsilon^{2}}\right)\left(\begin{array}{cc}
I & -I \\
-I & I
\end{array}\right)+o(1)\left(\begin{array}{ll}
I & 0 \\
0 & I
\end{array}\right)
$$

Indeed, besides the standard estimates for the second derivatives of $|x-y|^{2} / \varepsilon^{2}$, we also need to calculate those of $A(d(x)-d(y))^{2} / \varepsilon^{2}$ as well as those of

$$
\tilde{C}(x, t, y, s):=C_{\eta}(Q)(d(x)-d(y)) .
$$

Since the calculation for the former is similar to that for $|x-y|^{2} / \varepsilon^{2}$, we focus our attention on $\tilde{C}$. By direct calculations, we have

$$
\nabla^{2} \tilde{C}(x, t, y, s)=\sum_{i=1}^{3} D_{i}(Q)(d(x)-d(y))+\sum_{i=4}^{6} D_{i}(x, t, y, s),
$$

where

$$
\begin{gathered}
D_{1}(Q):=\frac{1}{4}\left(\begin{array}{ccc}
\nabla_{x}^{2} C_{\eta}(Q) & -\nabla_{x}^{2} C_{\eta}(Q) \\
-\nabla_{x}^{2} C_{\eta}(Q) & \nabla_{x}^{2} C_{\eta}(Q)
\end{array}\right), \quad D_{2}(Q):=\frac{2}{\varepsilon^{2}}\left(\begin{array}{cc}
\nabla_{x p}^{2} C_{\eta}(Q) & 0 \\
0 & -\nabla_{x p}^{2} C_{\eta}(Q)
\end{array}\right), \\
D_{3}(Q):=\frac{4}{\varepsilon^{4}}\left(\begin{array}{cc}
\nabla_{p}^{2} C_{\eta}(Q) & -\nabla_{p}^{2} C_{\eta}(Q) \\
-\nabla_{p}^{2} C_{\eta}(Q) & \nabla_{p}^{2} C_{\eta}(Q)
\end{array}\right)
\end{gathered}
$$

and

$$
\begin{gathered}
D_{4}(x, t, y, s)=\frac{1}{2}\left(\begin{array}{cc}
2 \nabla_{x} C_{\eta}(Q) \otimes \nabla d(x) & \nabla_{x} C_{\eta}(Q) \otimes(\nabla d(x)-\nabla d(y)) \\
\nabla_{x} C_{\eta}(Q) \otimes(\nabla d(x)-\nabla d(y)) & -2 \nabla_{x} C_{\eta}(Q) \otimes \nabla d(y)
\end{array}\right)^{\star}, \\
D_{5}(x, t, y, s)=\frac{2}{\varepsilon^{2}}\left(\begin{array}{cc}
2 \nabla_{p} C_{\eta}(Q) \otimes \nabla d(x) & -\nabla_{p} C_{\eta} \otimes(\nabla d(x)+\nabla d(y)) \\
-\nabla_{p} C_{\eta} \otimes(\nabla d(x)+\nabla d(y)) & 2 \nabla_{p} C_{\eta}(Q) \otimes \nabla d(y)
\end{array}\right)^{\star}, \\
D_{6}(x, t, y, s)=C_{\eta}(Q)\left(\begin{array}{cc}
\nabla^{2} d(x) & 0 \\
0 & -\nabla^{2} d(y)
\end{array}\right) .
\end{gathered}
$$

Here $A^{\star}$ denotes the symmetrized matrix of $A$, i.e., $A^{\star}=\left(A+A^{T}\right) / 2$.

We next verify that, evaluated at $Z_{\varepsilon}$, all terms in the sums appearing in (A.33) can be estimated by the right hand side of (A.32). First, by (A.7), (A.12) and (A.14), we have

$$
\begin{aligned}
& \left|D_{1}\left(Q_{\varepsilon}\right)\right|\left|d\left(x_{\varepsilon}\right)-d\left(y_{\varepsilon}\right)\right| \\
& \leq K\left(1+\frac{2}{\varepsilon^{2}}\left|x_{\varepsilon}-y_{\varepsilon}\right|\right)^{2}\left(\eta+\frac{2}{\varepsilon^{2}}\left|\left\langle\left(x_{\varepsilon}-y_{\varepsilon}\right), \nu\left(\frac{x_{\varepsilon}+y_{\varepsilon}}{2}\right)\right\rangle\right|\right)^{-1}\left|d\left(x_{\varepsilon}\right)-d\left(y_{\varepsilon}\right)\right| \\
& \leq K\left(1+\frac{2}{\varepsilon^{2}}\left|x_{\varepsilon}-y_{\varepsilon}\right|\right)^{2}\left(\frac{\varepsilon^{2}}{2}+\frac{1}{\eta} O\left(\left|x_{\varepsilon}-y_{\varepsilon}\right|^{2}\right)\right)=o(1) .
\end{aligned}
$$

Next, for the term with $D_{2}$, we use (A.8), (A.12) and (A.14) to similarly get

$$
\left|\nabla_{x p}^{2} C_{\eta}\left(Q_{\varepsilon}\right)\right|\left|d\left(x_{\varepsilon}\right)-d\left(y_{\varepsilon}\right)\right| \leq K\left(1+\frac{2}{\varepsilon^{2}}\left|x_{\varepsilon}-y_{\varepsilon}\right|\right)\left(\frac{\varepsilon^{2}}{2}+\frac{1}{\eta} O\left(\left|x_{\varepsilon}-y_{\varepsilon}\right|^{2}\right)\right)=o(\varepsilon),
$$


which implies that

$$
\left(d\left(x_{\varepsilon}\right)-d\left(y_{\varepsilon}\right)\right)\left\langle D_{2}\left(Q_{\varepsilon}\right)\left(w_{1} \oplus w_{2}\right), w_{1} \oplus w_{2}\right\rangle \leq \frac{1}{\varepsilon^{2}}\left|w_{1}-w_{2}\right|^{2}+o(1)\left(\left|w_{1}\right|^{2}+\left|w_{2}\right|^{2}\right)
$$

for all $w_{1}, w_{2} \in \mathbb{R}^{n}$. This shows that $D_{1}\left(Q_{\varepsilon}\right)\left(d\left(x_{\varepsilon}\right)-d\left(y_{\varepsilon}\right)\right)$ is bounded by the right hand side of (A.32). Moreover, since we can utilize (A.9) and (A.12) to deduce

$$
\frac{1}{\varepsilon^{4}}\left|\nabla_{p}^{2} C_{\eta}\left(Q_{\varepsilon}\right)\right|\left|d\left(x_{\varepsilon}\right)-d\left(y_{\varepsilon}\right)\right| \leq \frac{K}{\varepsilon^{2}}\left(\frac{1}{2}+\frac{1}{\varepsilon^{2}} O\left(\left|x_{\varepsilon}-y_{\varepsilon}\right|^{2}\right)\right),
$$

which yields

$$
D_{3}\left(Q_{\varepsilon}\right)\left(d\left(x_{\varepsilon}\right)-d\left(y_{\varepsilon}\right)\right) \leq O\left(\frac{1}{\varepsilon^{2}}\right)\left(\begin{array}{cc}
I & -I \\
-I & I
\end{array}\right) .
$$

The terms $D_{4}\left(Z_{\varepsilon}\right), D_{5}\left(Z_{\varepsilon}\right), D_{6}\left(Z_{\varepsilon}\right)$ can be similarly estimated. For instance, by direct computation, we have

$$
\begin{aligned}
& \left\langle D_{4}\left(Z_{\varepsilon}\right) w_{1} \oplus w_{2}, w_{1} \oplus w_{2}\right\rangle \\
& =\left\langle\nabla_{x} C_{\eta}\left(Q_{\varepsilon}\right) \otimes \nabla d\left(x_{\varepsilon}\right) w_{1}, w_{1}\right\rangle-\left\langle\nabla_{x} C_{\eta}\left(Q_{\varepsilon}\right) \otimes \nabla d\left(y_{\varepsilon}\right) w_{2}, w_{2}\right\rangle \\
& \quad+\left\langle\nabla_{x} C_{\eta}\left(Q_{\varepsilon}\right) w_{1}\right\rangle\left\langle\nabla d\left(x_{\varepsilon}\right)-\nabla d\left(y_{\varepsilon}\right), w_{2}\right\rangle+\left\langle\nabla_{x} C_{\eta}\left(Q_{\varepsilon}\right), w_{2}\right\rangle\left\langle\nabla d\left(x_{\varepsilon}\right)-\nabla d\left(y_{\varepsilon}\right), w_{1}\right\rangle
\end{aligned}
$$

for any $w_{1}, w_{2} \in \mathbb{R}^{n}$. By (A.4) and (A.14), we are led to

$$
\begin{aligned}
& \left\langle D_{4}\left(Z_{\varepsilon}\right) w_{1} \oplus w_{2}, w_{1} \oplus w_{2}\right\rangle \\
& \leq\left|\nabla_{x} C_{\eta}\left(Q_{\varepsilon}\right)\right||\nabla d(x)|\left|w_{1}-w_{2}\right|\left|w_{1}+w_{2}\right|+2 \nabla_{x} C_{\eta}\left(Q_{\varepsilon}\right)\left|\nabla d\left(x_{\varepsilon}\right)-\nabla d\left(y_{\varepsilon}\right)\right|\left(\left|w_{1}\right|^{2}+\left|w_{2}\right|^{2}\right) \\
& \leq o\left(\frac{1}{\varepsilon}\right)\left|w_{1}-w_{2}\right|\left|w_{1}+w_{2}\right|+o(1)\left(\left|w_{1}\right|^{2}+\left|w_{2}\right|^{2}\right) \\
& \leq O\left(\frac{1}{\varepsilon^{2}}\right)\left|w_{1}-w_{2}\right|^{2}+o(1)\left(\left|w_{1}\right|^{2}+\left|w_{2}\right|^{2}\right)
\end{aligned}
$$

as desired. We omit the tedious but analogous estimates for $D_{5}\left(Z_{\varepsilon}\right)$ and $D_{6}\left(Z_{\varepsilon}\right)$. In the estimate of $D_{6}\left(Z_{\varepsilon}\right)$, we need the Lipschitz continuity of $\nabla^{2} d$, which justifies our assumption on $C^{2,1}$ regularity of $\Omega$.

As the other terms in $\nabla^{2} \psi\left(Z_{\varepsilon}\right)$ can be estimated in a standard way, we conclude the proof of (A.32) by omitting the rest of the details.

4. Use of Crandall-Ishii lemma. Making use of Crandall-Ishii lemma, we see that there exist $X_{\varepsilon}, Y_{\varepsilon} \in \mathbb{S}^{n}$ such that

$$
\begin{gathered}
\left(\left(\nabla_{x} \psi\left(Z_{\varepsilon}\right), \partial_{t} \psi\left(Z_{\varepsilon}\right)\right), X_{\varepsilon}\right) \in \overline{\mathcal{P}}^{2,+} u\left(x_{\varepsilon}, t_{\varepsilon}\right), \\
\left(\left(-\nabla_{y} \psi\left(Z_{\varepsilon}\right),-\partial_{s} \psi\left(Z_{\varepsilon}\right)\right),-Y_{\varepsilon}\right) \in \overline{\mathcal{P}}^{2,-} v\left(y_{\varepsilon}, s_{\varepsilon}\right), \\
\left(\begin{array}{cc}
X_{\varepsilon} & O \\
O & Y_{\varepsilon}
\end{array}\right) \leq \nabla^{2} \psi\left(Z_{\varepsilon}\right)+\varepsilon^{2}\left(\nabla^{2} \psi\left(Z_{\varepsilon}\right)\right)^{2} .
\end{gathered}
$$

By (A.32), the last condition (A.36) implies that

$$
X_{\varepsilon}+Y_{\varepsilon} \leq o(1) .
$$

Since $u$ is a subsolution of (1.34) and (1.2), then (A.19) implies

$$
F\left(x_{\varepsilon}, \nabla_{x} \psi\left(Z_{\varepsilon}\right), X_{\varepsilon}\right) \leq 0
$$

even if $x_{\varepsilon} \in \partial \Omega$. Similarly, since $v$ is supersolution of (5.5) with (1.2) and (A.20) holds, we get

$$
F\left(y_{\varepsilon},-\nabla_{y} \psi\left(Z_{\varepsilon}\right),-Y_{\varepsilon}\right) \geq c .
$$


Subtracting (A.38) from (A.39), and applying (F3) and (A.37) together with the ellipticity of $F$, we obtain

$$
\begin{aligned}
c & \leq F\left(y_{\varepsilon},-\nabla_{y} \psi\left(Z_{\varepsilon}\right),-Y_{\varepsilon}\right)-F\left(x_{\varepsilon}, \nabla_{x} \psi\left(Z_{\varepsilon}\right), X_{\varepsilon}\right) \\
& \leq L_{F}\left(\left(1+\left|\nabla_{x} \psi\left(Z_{\varepsilon}\right)\right|+\left|\nabla_{y} \psi\left(Z_{\varepsilon}\right)\right|\right)\left|x_{\varepsilon}-y_{\varepsilon}\right|+\left|\nabla_{x} \psi\left(Z_{\varepsilon}\right)+\nabla_{y} \psi\left(Z_{\varepsilon}\right)\right|+o(1)\right) .
\end{aligned}
$$

By (A.29)-(A.31), we see that

$$
c \leq o(1)
$$

which is clearly a contradiction when $\varepsilon>0$ is sufficiently small.

\section{REFERENCES}

[1] E. S. Al-Aidarous, E. O. Alzahrani, H. Ishii, and A. M. M. Younas. Asymptotic analysis for the eikonal equation with the dynamical boundary conditions. Math. Nachr., 287(14-15):1563-1588, 2014.

[2] H. Amann and M. Fila. A Fujita-type theorem for the Laplace equation with a dynamical boundary condition. Acta Math. Univ. Comenian. (N.S.), 66(2):321-328, 1997.

[3] T. Antunović, Y. Peres, S. Sheffield, and S. Somersille. Tug-of-war and infinity Laplace equation with vanishing Neumann boundary condition. Comm. Partial Differential Equations, 37(10):1839-1869, 2012.

[4] S. N. Armstrong and C. K. Smart. An easy proof of Jensen's theorem on the uniqueness of infinity harmonic functions. Calc. Var. Partial Differential Equations, 37(3-4):381-384, 2010.

[5] S. N. Armstrong and C. K. Smart. A finite difference approach to the infinity Laplace equation and tug-of-war games. Trans. Amer. Math. Soc., 364(2):595-636, 2012.

[6] M. Bardi and I. Capuzzo-Dolcetta. Optimal control and viscosity solutions of Hamilton-Jacobi-Bellman equations. Systems \& Control: Foundations \& Applications. Birkhäuser Boston Inc., Boston, MA, 1997. With appendices by Maurizio Falcone and Pierpaolo Soravia.

[7] G. Barles. Fully nonlinear Neumann type boundary conditions for second-order elliptic and parabolic equations. J. Differential Equations, 106(1):90-106, 1993.

[8] G. Barles. Nonlinear Neumann boundary conditions for quasilinear degenerate elliptic equations and applications. J. Differential Equations, 154(1):191-224, 1999.

[9] G. Barles, H. Ishii, and H. Mitake. On the large time behavior of solutions of Hamilton-Jacobi equations associated with nonlinear boundary conditions. Arch. Ration. Mech. Anal., 204(2):515-558, 2012.

[10] I. Capuzzo-Dolcetta and P.-L. Lions. Hamilton-Jacobi equations with state constraints. Trans. Amer. Math. Soc., 318(2):643-683, 1990.

[11] F. Charro, J. García Azorero, and J. D. Rossi. A mixed problem for the infinity Laplacian via tug-ofwar games. Calc. Var. Partial Differential Equations, 34(3):307-320, 2009.

[12] P. Colli and T. Fukao. The Allen-Cahn equation with dynamic boundary conditions and mass constraints. Math. Methods Appl. Sci., 38(17):3950-3967, 2015.

[13] M. G. Crandall, H. Ishii, and P.-L. Lions. User's guide to viscosity solutions of second order partial differential equations. Bull. Amer. Math. Soc. (N.S.), 27(1):1-67, 1992.

[14] J.-P. Daniel. A game interpretation of the Neumann problem for fully nonlinear elliptic and parabolic equations. ESAIM: Control Optim. Calc. Var., 19(4):1109-1165, 2013.

[15] R. Denk, J. Prüss, and R. Zacher. Maximal $L_{p}$-regularity of parabolic problems with boundary dynamics of relaxation type. J. Funct. Anal., 255(11):3149-3187, 2008.

[16] C. M. Elliott, Y. Giga, and S. Goto. Dynamic boundary conditions for Hamilton-Jacobi equations. SIAM J. Math. Anal., 34(4):861-881, 2003.

[17] J. Escher. Nonlinear elliptic systems with dynamic boundary conditions. Math. Z., 210(3):413-439, 1992.

[18] J. Escher. Quasilinear parabolic systems with dynamical boundary conditions. Comm. Partial Differential Equations, 18(7-8):1309-1364, 1993.

[19] J. Escher. Smooth solutions of nonlinear elliptic systems with dynamic boundary conditions. In Evolution equations, control theory, and biomathematics (Han sur Lesse, 1991), volume 155 of Lecture Notes in Pure and Appl. Math., pages 173-183. Dekker, New York, 1994.

[20] L. C. Evans and R. F. Gariepy. Measure theory and fine properties of functions. Studies in Advanced Mathematics. CRC Press, Boca Raton, FL, 1992.

[21] L. C. Evans and P. E. Souganidis. Differential games and representation formulas for solutions of Hamilton-Jacobi-Isaacs equations. Indiana Univ. Math. J., 33(5):773-797, 1984. 
[22] M. Fila, K. Ishige, and T. Kawakami. Convergence to the Poisson kernel for the Laplace equation with a nonlinear dynamical boundary condition. Commun. Pure Appl. Anal., 11(3):1285-1301, 2012.

[23] M. Fila, K. Ishige, and T. Kawakami. Large-time behavior of small solutions of a two-dimensional semilinear elliptic equation with a dynamical boundary condition. Asymptot. Anal., 85(1-2):107-123, 2013.

[24] M. Fila, K. Ishige, and T. Kawakami. Large-time behavior of solutions of a semilinear elliptic equation with a dynamical boundary condition. Adv. Differential Equations, 18(1-2):69-100, 2013.

[25] M. Fila, K. Ishige, and T. Kawakami. The large diffusion limit for the heat equation with a dynamical boundary condition. preprint, ArXiv e-prints, 2018.

[26] M. Fila and P. Poláčik. Global nonexistence without blow-up for an evolution problem. Math. Z., 232(3):531-545, 1999.

[27] C. G. Gal and M. Grasselli. The non-isothermal Allen-Cahn equation with dynamic boundary conditions. Discrete Contin. Dyn. Syst., 22(4):1009-1040, 2008.

[28] Y. Giga and N. Hamamuki. Hamilton-Jacobi equations with discontinuous source terms. Comm. Partial Differential Equations, 38(2):199-243, 2013.

[29] Y. Giga and N. Hamamuki. On a dynamic boundary condition for singular degenerate parabolic equations in a half space. NoDEA Nonlinear Differential Equations Appl., 25(6):Art. 51, 39, 2018.

[30] Y. Giga and Q. Liu. A billiard-based game interpretation of the Neumann problem for the curve shortening equation. Adv. Differential Equations, 14(3-4):201-240, 2009.

[31] N. Hamamuki and Q. Liu. A game-theoretic approach to dynamic boundary problems for level-set curvature flow equations and applications. in preparation.

[32] H. Ishii. A simple, direct proof of uniqueness for solutions of the Hamilton-Jacobi equations of eikonal type. Proc. Amer. Math. Soc., 100(2):247-251, 1987.

[33] M. A. Katsoulakis. Viscosity solutions of second order fully nonlinear elliptic equations with state constraints. Indiana Univ. Math. J., 43(2):493-519, 1994.

[34] R. V. Kohn and S. Serfaty. A deterministic-control-based approach to motion by curvature. Comm. Pure Appl. Math., 59(3):344-407, 2006.

[35] R. V. Kohn and S. Serfaty. A deterministic-control-based approach to fully nonlinear parabolic and elliptic equations. Comm. Pure Appl. Math., 63(10):1298-1350, 2010.

[36] P.-L. Lions. Neumann type boundary conditions for Hamilton-Jacobi equations. Duke Math. J., 52(4):793-820, 1985.

[37] Q. Liu. Fattening and comparison principle for level-set equations of mean curvature type. SIAM J. Control Optim., 49(6):2518-2541, 2011.

[38] Q. Liu, A. Schikorra, and X. Zhou. A game-theoretic proof of convexity preserving properties for motion by curvature. Indiana Univ. Math. J., 65:171-197, 2016.

[39] H. Luiro, M. Parviainen, and E. Saksman. Harnack's inequality for $p$-harmonic functions via stochastic games. Comm. Partial Differential Equations, 38(11):1985-2003, 2013.

[40] J. J. Manfredi, M. Parviainen, and J. D. Rossi. An asymptotic mean value characterization for a class of nonlinear parabolic equations related to tug-of-war games. SIAM J. Math. Anal., 42(5):2058-2081, 2010 .

[41] J. J. Manfredi, M. Parviainen, and J. D. Rossi. An asymptotic mean value characterization for $p$ harmonic functions. Proc. Amer. Math. Soc., 138(3):881-889, 2010.

[42] M. Parviainen and E. Ruosteenoja. Local regularity for time-dependent tug-of-war games with varying probabilities. J. Differential Equations, 261(2):1357-1398, 2016.

[43] Y. Peres, O. Schramm, S. Sheffield, and D. B. Wilson. Tug-of-war and the infinity Laplacian. J. Amer. Math. Soc., 22(1):167-210, 2009.

[44] Y. Peres and S. Sheffield. Tug-of-war with noise: a game-theoretic view of the p-Laplacian. Duke Math. J., 145(1):91-120, 2008.

[45] E. Ruosteenoja. Local regularity results for value functions of tug-of-war with noise and running payoff. Adv. Calc. Var., 9(1):1-17, 2016.

[46] H. M. Soner. Optimal control with state-space constraint. I. SIAM J. Control Optim., 24(3):552-561, 1986.

[47] H. M. Soner. Optimal control with state-space constraint. II. SIAM J. Control Optim., 24(6):11101122, 1986.

[48] J. Sprekels and H. Wu. A note on parabolic equation with nonlinear dynamical boundary condition. Nonlinear Anal., 72(6):3028-3048, 2010.

[49] J. L. Vázquez and E. Vitillaro. Heat equation with dynamical boundary conditions of reactive type. Comm. Partial Differential Equations, 33(4-6):561-612, 2008. 
(N. Hamamuki) Department of Mathematics, Hokkaido University, Kita 10, Nishi 8, KitaKu, SAPporo, HoKKAIDO 060-0810, JAPAN. E-mail address: hnao@math.sci.hokudai.ac.jp

(Q. Liu) Department of Applied Mathematics, Faculty of Science, Fukuoka University, FUKUOKA 814-0180, JAPAN. E-mail address: qingliu@fukuoka-u.ac.jp 